\title{
Inflow Generated X-ray Corona Around Supermassive Black Holes and Unified Model for X-ray Emission
}

\author{
Lile Wang $^{1}$ and Renyue Cen ${ }^{2}$
}

\begin{abstract}
Three-dimensional hydrodynamic simulations, covering the spatial domain from hundreds of Schwarzschild radii to $2 \mathrm{pc}$ around the central supermassive black hole of mass $10^{8} M_{\odot}$, with detailed radiative cooling processes, are performed. Generically found is the existence of a significant amount of shock heated, high temperature $\left(\geq 10^{8} \mathrm{~K}\right)$ coronal gas in the inner $\left(\leq 10^{4} r_{\text {sch }}\right)$ region. It is shown that the composite bremsstrahlung emission spectrum due to coronal gas of various temperatures are in reasonable agreement with the overall ensemble spectrum of AGNs and hard X-ray background. Taking into account inverse Compton processes, in the context of the simulation-produced coronal gas, our model can readily account for the wide variety of AGN spectral shape, which can now be understood physically. The distinguishing feature of our model is that X-ray coronal gas is, for the first time, an integral part of the inflow gas and its observable characteristics are physically coupled to the concomitant inflow gas. One natural prediction of our model is the anti-correlation between accretion disk luminosity and spectral hardness: as the luminosity of SMBH accretion disk decreases, the hard X-ray luminosity increases relative to the UV/optical luminosity.
\end{abstract}

Subject headings: accretion, accretion disks — galaxies: active — galaxies: nuclei — quasars: general - X-rays: galaxies — methods: numerical

\section{Introduction}

According to the commonly accepted paradigm, a luminous Active Galactic Nucleus (AGN) has a supermassive black hole at the center embedded in an equatorial accretion disc. The hard non-thermal emission is generally thought to arise in a hot gas above the accretion disc (e.g., Elvis 2000).

Hot accretion flow based on the vertical one-zone approximation is shown to possibly play a role, coming in a variety of flavors, such as advection-dominated accretion flow, convectiondominated accretion flow, and advection-dominated inflow-outflow solution (e.g., Ichimaru 1977;

\footnotetext{
${ }^{1}$ Princeton University Observatory, Princeton, NJ 08544; lilew@astro.princeton.edu

2Princeton University Observatory, Princeton, NJ 08544; cen@astro.princeton.edu
} 
Narayan \& Yi 1994; Blandford 1999; Igumenshchev \& Abramowicz 2000), some of which can reasonably fit the observed X-ray spectra of low-luminosity AGNs (e.g., Narayan et al. 1998). The concurrent soft and hard X-ray components observed in ordinary AGNs and the high X-ray luminosity are not well modeled by the one-zone hot-flow models. A composite disk-corona structure may be required. The generally accepted model is that UV/optical seed photons from the disc powered by accretion power are Compton upscattered by hot thermal electrons in the corona. Such a physical process leads to a power-law spectrum extending to energies determined by the electron temperature in the hot corona. The power-law index is a function of the plasma temperature and optical depth (e.g., Sunyaev \& Titarchuk 1980; Haardt \& Maraschi 1991; Haardt et al. 1994; Pietrini \& Krolik 1995; Veledina et al. 2011). In addition, the primary $\mathrm{X}$-ray radiation in turn illuminates the disc and it is partly reflected towards the observer's line of sight, often in fluorescence from near-neutral (for iron) material of the disc, that has been observed (e.g., Fabian et al. 1989; Tanaka et al. 1995; Reynolds \& Nowak 2003).

Since the spectrum depends on the optical depth and the temperature of the hot gas, spectral shape can be reproduced by some combinations of these parameters. This successful model has a major missing element. That is, we do not know what heats the corona plasma to temperatures as high as $10^{9} \mathrm{~K}$. Heating via magnetic field reconnection (e.g., Di Matteo 1998; Miller \& Stone 2000; Liu et al. 2002, 2003; Ohsuga \& Mineshige 2011) may be a relevant process, perhaps operating in a fashion similar to solar corona.

Here we explore the dynamics of gas inflows using three-dimensional hydrodynamic simulations to attempt to construct an alternative model. Our approach is to survey the gas feeding conditions at large scales and examine how they affect the distribution of shock heated gas powered by gravitational energy of inflow gas. Our simulations show that hot gas of temperatures often exceeding $10^{9} \mathrm{~K}$, resembling the purported corona, can be naturally produced in the inner regions of $\lesssim 3 \times 10^{3} r_{\text {sch }}$, with hotter gas being closer to the black hole. The composite bremsstrahlung emission alone from the hot gas with a range of temperatures may be able to account for a significant fraction, if not most, of the X-ray emission observed in high (optical/UV) luminosity AGNs, and of the hard X-ray background. We combine the simulation results with a treatment of the inverse Compton process to show that the observed anti-correlation between the optical/UV luminosity and the hardness of the optical-X-ray spectral index is naturally explained. The physical origin is of self-regulation in nature. At high optical-UV luminosity of the AGN, the high inverse Compton cooling rate renders the inner region devoid of significant hot coronal gas. Thus, the inverse Compton optical depth and overall temperature of the coronal gas are low. The resulting, relatively weak X-ray emission in this case is primarily contributed by bremsstrahlung process. At low optical-UV luminosity, the low inverse Compton cooling rate results in a more centrally peaked and overall higher inverse Compton optical and coronal gas temperature, which in turn yield harder and comparatively higher inverse Compton emission that is now dominant. We show that our model explains remarkably well the observed range of optical-X-ray spectral index for Eddington ratios ranging from $10^{-3}$ to 0.3 . This paper is structured as follows. Section 2 describes the setup of our sim- 
ulations and methods of analysis. Results are presented in Section 3, followed by conclusions in Section 4.

\section{Computational Methods}

\subsection{Hydrodynamic Code}

We perform simulations of gas flows around supermassive black holes with the widely used adaptive mesh refinement (AMR) Eulerian hydro code, Enzo (Bryan et al. 2014). Our simulation domain covers a three dimensional cubic box with side length $L_{\text {box }}=4 \mathrm{pc}$, in which Cartesian coordinates are initially established. The root grid (i.e., the base level) is $128^{3}$, upon which additional refinement levels may be applied. We find, upon inspecting several running examples, that the "effective" regions in which refinement should be applied turns out to be relatively invariant. Thus, we initially lay down a set of statically refined grid with five levels, with a refinement factor of two for each level, detailed in Table 1. We also run one simulation run with six levels of refinement to test numerical convergence.

We solve the hydrodynamics equations with Newtonian gravity.

$$
\begin{aligned}
\frac{\partial \rho}{\partial t}+\nabla \cdot(\rho \mathbf{v}) & =0 \\
\frac{\partial \mathbf{v}}{\partial t}+(\mathbf{v} \cdot \nabla) \mathbf{v}+\frac{1}{\rho} \nabla p+\nabla \Phi & =0 \\
\frac{\partial e}{\partial t}+(\mathbf{v} \cdot \nabla) e+\frac{p}{\rho} \nabla \cdot \mathbf{v} & =\Gamma-\Lambda, \\
\nabla^{2} \Phi & =4 \pi G \rho
\end{aligned}
$$

where $\rho, p, e, \mathbf{v}$ and $\Phi$, indicate mass density, thermal pressure, specific internal energy per unit mass,velocity vector and gravitational potential, respectively; $G$ is the gravitational constant. There are several hydro modules implemented in ENZO. We use the most robust ZEUS hydro solver, which incorporates artificial viscosity to capture shocks, producing correct

Table 1: Geometry of refinement regions. Those are rectangular regions in $x \times y \times z$ in units of $L_{\mathrm{box}}$, centered at the box center.

\begin{tabular}{cccc}
\hline Region No. & Level & Shape & Dimension (in $L_{\text {box }}$ ) \\
\hline 1 & 1 & rectangular & $0.5 \times 0.5 \times 0.05$ \\
2 & 2 & rectangular & $0.16 \times 0.16 \times 0.02$ \\
3 & 3 & rectangular & $0.08 \times 0.08 \times 0.01$ \\
4 & 4 & rectangular & $0.04 \times 0.04 \times 0.01$ \\
5 & 5 & rectangular & $0.04 \times 0.04 \times 0.01$ \\
6 & 6 & rectangular & $0.04 \times 0.04 \times 0.01$ \\
\hline
\end{tabular}


Rankine-Hugoniot conditions across shock fronts (see also Stone \& Norman 1992). We note here that for the energy equation in Equation $1, \Lambda$ and $\Gamma$ are the total of radiative cooling and heating rates, respectively.

The radiative cooling term $\Lambda$ includes all cooling processes from a primordial gas and metal cooling processes assuming solar metallicity. The heating term mainly consists of photoionization heating of all species in the gas and Compton heating. This heating term is not treated in this work (i.e. $\Gamma=0$ ) due to lack of self-consistent calculation of radiative transfer, which thus has enhanced cooling of gas the simulations. Thus, our findings of very hot gas in the central region is likely on the conservative side.

\subsection{Setup}

In each model, a supermassive black hole of mass $M_{\mathrm{BH}}=10^{8} M_{\odot}$ is placed at the center of the box, with a gravitational acceleration

$$
\mathbf{g}=-\frac{G M_{\mathrm{BH}} \hat{\mathbf{r}}}{r_{0}^{2}}, \quad r_{0}=\left\{\begin{array}{l}
r, r>a ; \\
a, r \leq a ;
\end{array}\right.
$$

where $a$ is the core radius, which may be varied; $r$ is the distance from the black hole, and $\hat{\mathbf{r}}$ is the unit vector pointing at the cell in question from the $\mathrm{SMBH}$. This softening radius, set to be significantly larger than the hydro resolution, allows us to gauge how gravitational heating affects the gas density and temperature structure and also (easily) avoid numerical singularity near the black hole.

An accretion disk of mass $\sim 10^{7} M_{\odot}$ surrounds the SMBH. Rather than placing the disk by hand, it is built by feeding gas with the same pattern (angular momentum distribution) but with a higher feeding rate for about $\sim 2 \times 10^{4} \mathrm{yr}$, after which we turn off gas feeding at the boundary so that the disk structure can relax for $\sim 3 \times 10^{4} \mathrm{yr}$. This point, at the end of the disk relaxation, marks time $t=0$ for each of the simulations. We do not include self-gravity of the gas disk and other gas. Realistic gas feeding rate starts at $t=0$. We use data outputs at $t>10^{4} \mathrm{yr}$ only, to allow for the gas feeding pattern to reach a statistically steady state, noting that the free fall time at the boundary of the box is $5.7 \times 10^{3} \mathrm{yr}$. Data outputs in $t=10^{4}-2 \times 10^{4} \mathrm{yr}$ are used for all subsequent analysis, with a frequency of one output every $10 \mathrm{yr}$.

At the outer boundaries, gas inflows are fed through a fraction of random grid cells. The rest of the cells are marked as "free" (or "Dirichlet") boundary. Boundary feedings has specific fixed value of total mass inflow rate, denoted as $\dot{M}_{\text {feed }}$. Gas feeding is through 10 percent of the randomly chosen "pixels" of the feeding faces. We do not feed gas from the top and bottom faces of the simulation box. Among the four side faces, we vary the number of faces for feeding to assess the effects of feeding (a)symmetries. Thus, the feeding rate at a given 
pixel on the side faces is

$$
\dot{M}_{\text {feed, pixel }}=\frac{\dot{M}_{\text {feed }}}{0.1 N_{\text {feed }}}\left(\frac{\delta l}{L_{\text {box }}}\right)^{2} \Theta(0.1-X),
$$

where $\delta l=L_{\mathrm{box}} / 128$ is the pixel size at the boundary, $N_{\text {feed }}=1,2,4$ is the number of side faces that is fed through, $X$ is a random variable obeying the uniform distribution $U(0,1)$. $\Theta(x)$ is the Heaviside step function, where $\Theta(x)=1$ for all $x>0$ and $\Theta(x)=0$ for all $x<0$. This randomness is introduced to emulate stochastic feeding. However, in our simulations, we find that the result is little changed, in terms of central coronal density and temperature profile and hence radiative characteristics, if we change the fraction through which the gas is fed, or totally remove this random feeding scheme and adopt a steady feeding across chosen boundary cells.

The inflow gas fed through the outer boundary obeys the following specific angular momentum distribution:

$$
\frac{\mathrm{d} \dot{M}_{\text {feed }}(j)}{\mathrm{d} j}= \begin{cases}\frac{\dot{M}_{\text {feed }}}{2\langle j\rangle}, & 0<j<2\langle j\rangle ; \\ 0, & \text { elsewhere }\end{cases}
$$

with the expectation value $\langle j\rangle$ that may vary for different models. Note that such an angular momentum distribution would correspond to the classic Mestel (1963) disk, if allowed to settle on its own self-gravity. Such a distribution is an excellent approximation to results from realistic, high resolution $(\leq 0.1 \mathrm{pc})$ simulations (Hopkins \& Quataert 2010, 2011).

The set of simulations performed is listed in Table 2. Run " 0 " is our fiducial run with $\dot{M}_{\text {feed }, 0} \simeq 8.1 M_{\odot} / \mathrm{yr}$, the expectation value of the angular momentum distribution $j_{0}$ where the value of $j_{0}$ is such that gas with $j_{0}$ is rotationally supported when reaching $r \sim 0.4 \mathrm{pc}$, gravitational softening length $a=a_{0} \equiv 1250 r_{\text {sch }}$ in Equation (2) where $r_{\text {sch }}$ is the Schwartzchild radius of the $\mathrm{SMBH}$ at the center $\left(r_{\text {sch }}=1.97 \mathrm{AU}\right)$. The 22 additional simulations are performed by varying one of the five parameter at a time. Specifically, we vary the feeding angular momentum for 8 additional runs (Runs 1, 2, 3 at high feeding rate $\dot{M}_{\text {feed }} / \dot{M}_{\text {feed }, 0}=1$ with $\langle j\rangle / j_{0}=0.1,0.01,0.001$; Runs 9,10 at low feeding rate $\dot{M}_{\text {feed }} / \dot{M}_{\text {feed, } 0}=0.01$ with $\langle j\rangle / j_{0}=0.1,0.01$; Runs $16,17,18$ at high feeding rate $\dot{M}_{\text {feed }} / \dot{M}_{\text {feed, } 0}=1$ with two feeding faces and $\langle j\rangle / j_{0}=0.5,2,5$ ), the feeding rate for 10 additional runs (Runs $4,5,6,7,8$ at low angular momentum $\langle j\rangle / j_{0}=0.001$ with $\dot{M}_{\text {feed }} / \dot{M}_{\text {feed }, 0}=0.1,0.01,0.005,0.002,0.001$; Runs $11,12,13,14,15$ at high angular momentum $\langle j\rangle / j_{0}=1$ with feeding rate $\dot{M}_{\text {feed }} / \dot{M}_{\text {feed }, 0}=$ $10,3,1,0.3,0.1$ and two feeding faces), the gravitational softening radius for two additional runs (Runs 19, 20 with $a / a_{0}=0.5,0.25$ ), the number of feeding faces for one additional run (Run 21) and finally an additional run (Run 21) to vary the resolution. 
Table 2: Parameters of simulations.

\begin{tabular}{cccccc}
\hline Model No. & $\dot{M}_{\text {feed }} / \dot{M}_{\text {feed, } 0}$ & $\langle j\rangle / j_{0}$ & $a / a_{0}$ & Feeding face & Static Refinement Levels \\
\hline 0 & 1 & 1 & 1 & 1 & 5 \\
1 & 1 & 0.1 & 1 & 1 & 5 \\
2 & 1 & 0.01 & 1 & 1 & 5 \\
3 & 1 & 0.001 & 1 & 1 & 5 \\
4 & 0.1 & 0.001 & 1 & 1 & 5 \\
5 & 0.01 & 0.001 & 1 & 1 & 5 \\
6 & 0.005 & 0.001 & 1 & 1 & 5 \\
7 & 0.002 & 0.001 & 1 & 1 & 5 \\
8 & 0.001 & 0.001 & 1 & 1 & 5 \\
9 & 0.01 & 0.1 & 1 & 1 & 5 \\
10 & 0.01 & 0.01 & 1 & 1 & 5 \\
11 & 10 & 1 & 1 & 2 & 5 \\
12 & 3 & 1 & 1 & 2 & 5 \\
13 & 1 & 1 & 1 & 2 & 5 \\
14 & 0.3 & 1 & 1 & 2 & 5 \\
15 & 0.1 & 1 & 1 & 2 & 5 \\
16 & 1 & 0.5 & 1 & 2 & 5 \\
17 & 1 & 2 & 1 & 2 & 5 \\
18 & 1 & 5 & 1 & 2 & 5 \\
19 & 1 & 1 & 0.5 & 2 & 5 \\
20 & 1 & 1 & 0.25 & 2 & 5 \\
21 & 1 & 1 & 1 & 4 & 5 \\
22 & 1 & 1 & 1 & 2 & 5 \\
\hline
\end{tabular}




\subsection{Post-Simulation Analysis}

The X-ray emissivity by bremsstrahlung process (denoted by subscript "ff") is (e.g. Draine 2011)

$$
\begin{aligned}
j_{\mathrm{ff}} & =5.44 \times 10^{-41} \mathrm{erg} \mathrm{cm}^{-3} \mathrm{~s}^{-1} \mathrm{~Hz}^{-1} \mathrm{sr}^{-1} \\
& \times g_{\mathrm{ff}} T_{4}^{-1 / 2}\left[\frac{n_{i} n_{e}}{\left(1 \mathrm{~g} \mathrm{~cm}^{-3}\right)^{2}}\right] Z_{i}^{2} \mathrm{e}^{-h \nu / k T},
\end{aligned}
$$

where $n_{i}$ and $n_{e}$ are the number density of ionized particles, $T_{4}=\left(T / 10^{4} \mathrm{~K}\right)$ where $T$ is the plasma temperature, $Z_{i}$ is the effective charge number of the ions, and $g_{\mathrm{ff}}$ is the Gaunt factor,

$$
g_{\mathrm{ff}} \simeq \ln \left\{\mathrm{e}+\exp \left[5.96-\frac{\sqrt{3}}{\pi} \ln \left(Z \nu_{9} T_{4}^{-3 / 2}\right)\right]\right\},
$$

in which $\nu_{9}=\left(\nu / 10^{9} \mathrm{~Hz}\right)$. Given the simulation output data, Equations (5) and (6) will be applied to calculate the spectra of free-free emission. We note here that the electron-electron bremsstrahlung is safely ignored, since this only under-estimates the bremsstrahlung emission by a few per cent in the $[10 \mathrm{keV}, 100 \mathrm{keV}]$ regime, at electron temperature $T_{e} \sim 10^{9} \mathrm{~K}$ (see also Haug 1975). We obtain total emission in different energy bands by integrating Equation (5) through specific energy ranges.

The disk is distinguished from coronal region. We procedurally exclude the disk by weighting the the computed quantity in question by a factor $\mathrm{e}^{-\tau / 2}$, where $\tau$ is the optical depth of the "pixel". Using a different weighting factor $\mathrm{e}^{-\tau}$ does not produce materially different results. For example, we find that the difference in total X-ray emission differs by about 3 per cent between the two weighting schemes. We also tested an additional criterion, making use of the fact that the disk gas usually obey $v_{t}>v_{r}$ (i.e., the tangential velocity being greater than the radial velocity). This additional criterion, together with the optical depth one, gives a composite weighting factor $\Theta\left(v_{r}-v_{t}\right) \mathrm{e}^{-\tau / 2}$, where $\Theta(x)$ is the Heaviside theta function with argument $x$. The overall results using this composite weighting factor are very similar to that using $e^{-\tau / 2}$ factor alone.

\section{Results}

\subsection{Existence of Generic Gravitational Shock Heated Coronal Gas}

Figure 1 shows the gas density (in $\mathrm{g} \mathrm{cm}^{-3}$, panel a), radial velocity (panel b), tangential velocity magnitude (panel c), temperature (panel d), X-ray emissivity in the $[1 \mathrm{keV}, 10 \mathrm{keV}]$ band (panel e) and in the $[10 \mathrm{keV}, 100 \mathrm{keV}]$ band (panel f), total pressure (thermal pressure plus ram pressure, panel g), Mach number (panel h), and ratio of kinetic and entropic energy (calculated with Sackur-Tetrode entropy, panel i), for a slice with one-cell thickness of size $10^{4} r_{\text {sch }}$ cutting through the center and perpendicular to the disk from the fiducial simulation. It 

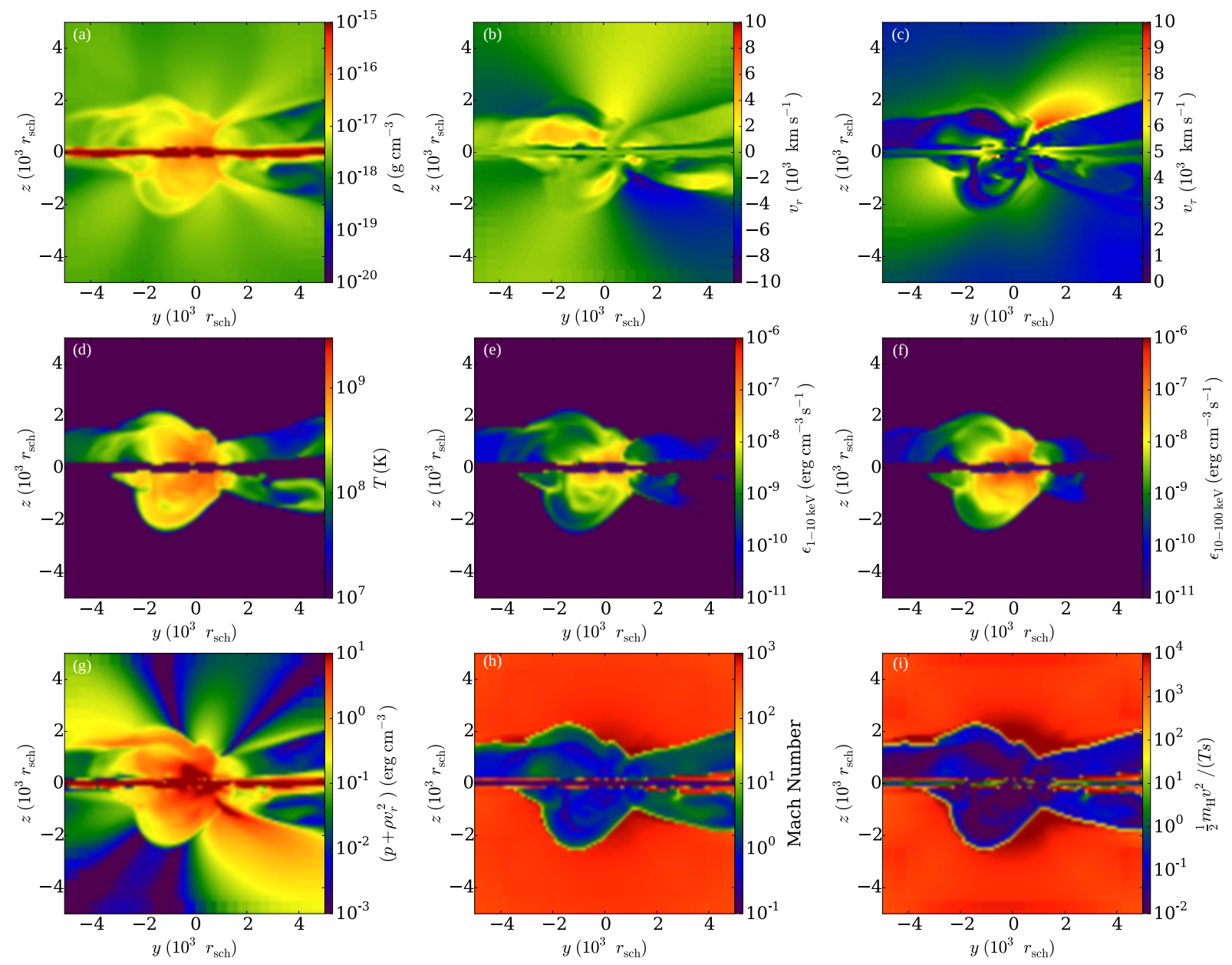

Fig. 1. - Shows the gas density (in $\mathrm{g} \mathrm{cm} \mathrm{cm}^{-3}$, panel a), radial velocity $\left(v_{r}\right.$ in $10^{3} \mathrm{~km} \mathrm{~s}^{-1}$, panel b), magnitude of tangential velocity $\left(v_{\tau}\right.$ in $10^{3} \mathrm{~km} \mathrm{~s}^{-1}$, panel c), temperature (in Kelvin, panel d), integrated X-ray emissivity (in erg $\mathrm{cm}^{-3} \mathrm{~s}^{-1}$ ) in the $[1 \mathrm{keV}, 10 \mathrm{keV}]$ band (panel e) and in the $[10 \mathrm{keV}, 100 \mathrm{keV}]$ band (panel f), total pressure (the sum of gas pressure $p$ and radial ram pressure $\rho v_{r}^{2}$, in erg $\mathrm{cm}^{-3}$, panel $\mathrm{g}$ ), Mach number (dimensionless, panel $\mathrm{h}$ ), and ratio between single particle bulk motion kinetic energy $\left(m_{\mathrm{H}} v^{2} / 2\right)$ and $T s$ (temperature times single particle Sackur-Tetrode entropy) (dimensionless, panel i), for a slice of size $10^{4} r_{\text {sch }}$ cutting through the center and perpendicular to the disk, from the fiducial simulation (Run 0 in Table 2).

is noted that there is a cold ( $\lesssim 10^{4} \mathrm{~K}$, below the range of the color map) disk at the equatorial plane due to its high density and short cooling time. Since we do not properly treat the viscous energy dissipation in the disk as well as the radiative losses, the temperature of the disk is not correct. Nevertheless, our calculation of X-ray emission in the corona is not contingent upon a realistic treatment of the dense disk. It is possible that, due to the disk colder than it should be, some coronal gas will be more prone to sticking/accreting to the disk due to an under-estimate of vertical pressure gradient near the disk. Thus, it is likely that we have somewhat underestimated the hot coronal gas due to this mis-treatment of accretion disk. The contrasting temperature, density and kinematics allow us to easily separate the corona material from the 
disk.

The coronal regions, above and below the disk, are easily distinguished from the disk by their high temperatures $10^{8-9} \mathrm{~K}$ and lower density. The coronal regions of hot gas are seen to occupy the central regions of size a few times $10^{3} r_{\text {sch. }}$. While we will show that the dynamics of the coronal region depends on the boundary conditions (feeding rate, angular momentum distribution, feeding pattern, etc), our simulation box is more than ten times larger than the coronal regions of interest and its size is thus large enough to not impact the properties of the coronal regions. All our results are based on time snapshots after $\gtrsim 10^{4} \mathrm{yr}$, compared to the free fall time of $\sim 800$ yr at $2 \times 10^{4} r_{\text {sch }}$, the coronal regions with hard X-ray emission is fully relaxed and basically in a statistically steady state.

The only heating mechanisms that are allowed here in the simulation are shocks and adiabatic compression. Boundaries of heated regions are seen to be quite sharp, suggesting that the main heating mechanism is shock heating. In order to further confirm this, we show in panels (g), (h) and (i) in Figure 1 the quantities that are pertinent to shocks. Panel (h) shows clearly a large jump in Mach number across the shock heated "surface". Shock dissipation is also consistent with panel (i) showing a drastic increase inward of the entropy across the shocked surface. Note that compression heating conserves entropy. The total pressure (thermal + ram pressure) (panel g) demonstrates dynamically that compression does not play a role, since the pressure increases inward, instead of outward. In general, there is a trend that higher temperature gas is more centrally concentrated than lower temperature gas. These features are generic among all simulations with varying feeding parameters at the boundary.

Figure 2 shows the radial distribution profiles of density (top panel), bremsstrahlung emissivity (middle panel) and emissivity-weighted hydrogen density (bottom panel) of the coronal gas in three X-ray bands. Note that we exclude the disk when making these plots. In all panels, we confirm the visual evidence seen in Figure 1 that the hard X-ray emission is centrally concentrated. For both $[1 \mathrm{keV}, 10 \mathrm{keV}]$ and $\left[10 \mathrm{keV}, 10^{2} \mathrm{keV}\right]$ bands, the $\mathrm{X}$-ray emission mainly come from the central $10^{4} r_{\text {sch }}$, while for the $\left[10^{2} \mathrm{keV}, 10^{3} \mathrm{keV}\right]$ band the central $10^{3} r_{\text {sch }}$ dominates. Significant fluctuations in X-ray emission on the time scale of (at least as short as) $10 \mathrm{yr}$ is seen, as indicated by the errorbars, suggesting that the $\mathrm{X}$-ray emitting regions are not static, with higher temperature gas exhibiting larger amplitude temporal fluctuations than lower temperature gas. To place things in a more quantitative context, the mean bremsstrahlung X-ray luminosity is $1.65 \times 10^{43} \mathrm{erg} \mathrm{s}^{-1}$ for $E>1 \mathrm{keV}$, and $1.17 \times 10^{43} \mathrm{erg} \mathrm{s}^{-1}$ for $E>10 \mathrm{keV}$, compared to the Eddington luminosity of $L_{\mathrm{Edd}}\left(10^{8} M_{\odot}\right) \simeq 1.3 \times 10^{46} \mathrm{erg} \mathrm{s}^{-1}$ for a $10^{8} M_{\odot}$ black hole.

It is useful to have a basic understanding of the gas temperature. If one equates the thermal energy $(3 k T / 2)$ of a particle around the SMBH to the free-fall energy at radius $r$, one finds that,

$$
T_{\text {vir }}=6.3 \times 10^{8} \mathrm{~K}\left(\frac{r}{3 \times 10^{3} r_{\mathrm{sch}}}\right)^{-1} .
$$

It is easy to see that the gas temperature obtained from the simulation is in good accordance 


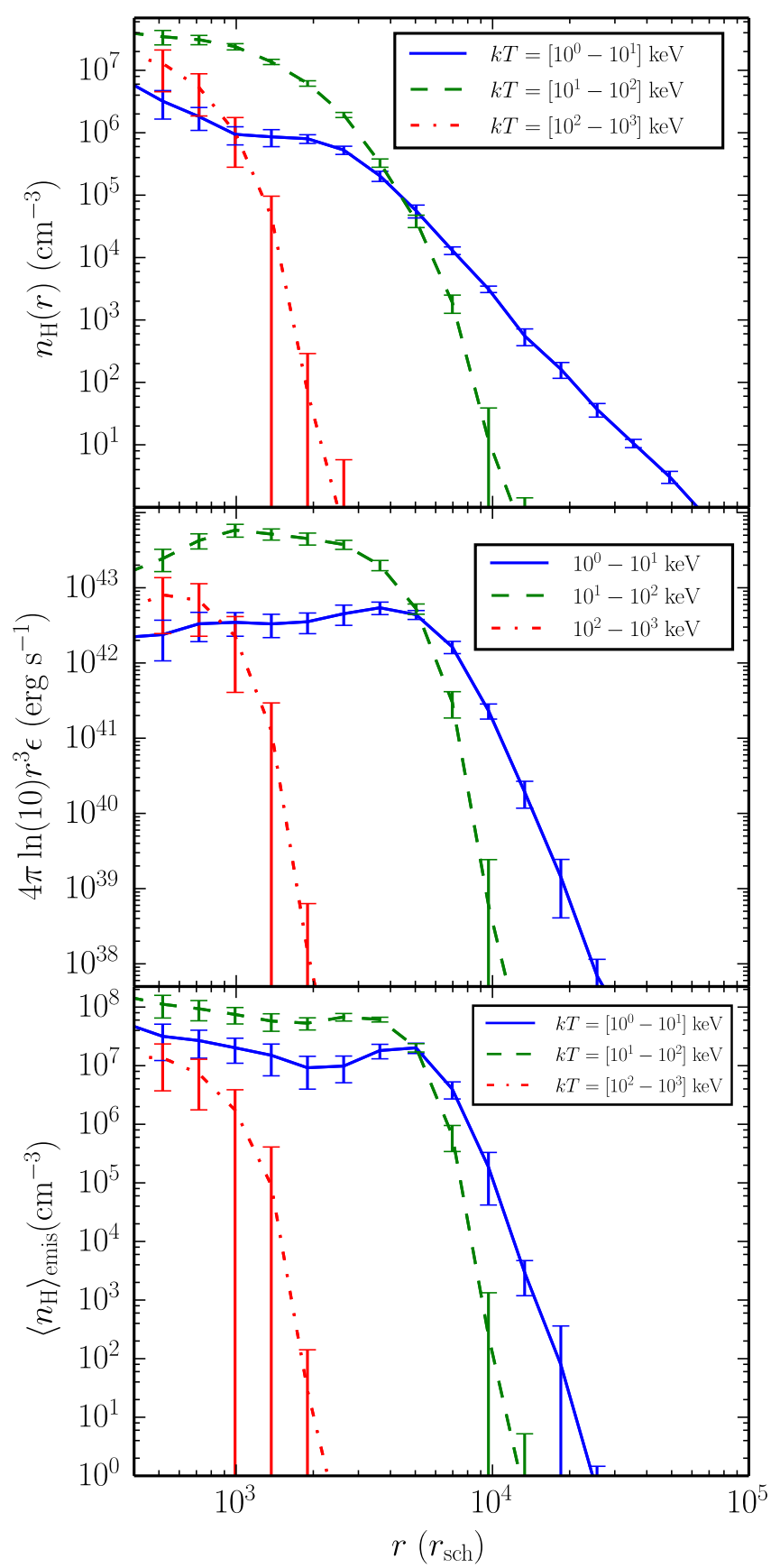

Fig. 2.- Top panel shows the radial profile of density, averaged over all $4 \pi$ solid angle, in three X-ray energy bands: [1 keV, $10 \mathrm{keV}]$ (blue solid), [10 keV, $\left.10^{2} \mathrm{keV}\right]$ (blue dashed), $\left[10^{2} \mathrm{keV}, 10^{3} \mathrm{keV}\right]$ (red dot-dashed). Middle panel shows the radial distributions of luminosity, emitted by bremsstrahlung processes in the corona. Note that we have multiplied the emissivity $\epsilon$ by $4 \pi \ln (10) r^{3}$ so that the area below each line is the luminosity. Bottom panel shows the radial profile of emissivity-weighted hydrogen density for the three respective bands. We use 100 snapshots over a time period of $10^{3}$ yr to compute the distribution, with the errorbars shown indicating the inter-quartiles. 
with this estimate, after infalling gas being shock heated. From panel (i) of Figure 1, we clearly observe that the gas preserve its bulk motion kinetic energy above the shock surface, at which kinetic energy is thermalized. Needless to say, the ultimate energy source is gravitational. The amount of feeding gas reaching various radii will depend on the physical conditions and dynamics of the gas, with varying amounts of gas at varying radii cooling out from the inflow to join the disk. Therefore, the intensity of $\mathrm{X}$-ray emission out of this dynamic mechanism will likely be determined by the amount of radial mass flux into the region of $R \lesssim 3 \times 10^{3} r_{\text {sch }}$ if the gas cooling time is shorter than the dynamical time, by the amount of hot gas accumulated over the cooling time if the cooling time is longer than the dynamical time.

Coulomb collisions are the main physical mechanism that equilibrates electron and ion temperatures. The equilibrating timescale at radius $r$ for a fully ionized medium (Spitzer 1962), comprised of electrons, protons, and $\mathrm{He} \mathrm{III,} \mathrm{is}$

$$
\begin{aligned}
t_{\mathrm{ei}} & \simeq 2 \times 10^{-2} t_{\mathrm{dyn}}\left(\frac{r}{3 \times 10^{3} r_{\mathrm{sch}}}\right)\left(\frac{T_{e}}{10^{8} \mathrm{~K}}\right) \\
& \times\left(\frac{n_{\mathrm{H}}}{10^{7} \mathrm{~cm}^{-3}}\right)^{-1}\left(\frac{\ln \Lambda}{40}\right)^{-1},
\end{aligned}
$$

where

$$
t_{\mathrm{dyn}} \simeq 10^{3} \mathrm{~s}\left(\frac{M_{\mathrm{BH}}}{10^{8} M_{\odot}}\right)\left(\frac{r}{r_{\mathrm{sch}}}\right)^{3 / 2},
$$

is the free-fall time (viz. the dynamical time scale), $T_{e}$ the notional electron temperature, $n_{\mathrm{H}}$ the hydrogen number density, $\Lambda$ the Coulomb logarithm. Given the typical density $n_{\mathrm{H}} \sim 10^{6}-$ $10^{7} \mathrm{~cm}^{-3}$, as is seen in Figure 2, we conclude that treating electrons and ions as a single temperature fluid is a reasonable approximation. This ensures that our calculation of $\mathrm{X}$-ray emissions, using the shock heating temperature, from both the free-free process and inverse Compton (see below) processes, is valid.

\subsection{Dependence of Coronal Gas on Boundary Feeding Conditions}

We now show the impact of different boundary feeding conditions on the concerned coronal gas. We first examine how the basic physical quantities in the X-ray emitting regions depend on the gas feeding conditions at the boundary, utilizing the set of simulations listed in Table 2. As indicated by simulation results so far, the gas flowing inward is subject to both heating and cooling processes, which, together with dynamical interactions, such as angular momentum exchanges among gas, determine the amount of mass flux into the inner regions. Those in turn determine the $\mathrm{X}$-ray emission at various energy bands. Figure 3 shows the radial mass flux (first row; positive for inflow), the bremsstrahlung cooling time in units of free fall time (second row), gas temperature (third row), and gas number density (bottom row). In all panels, the cold dense disk is excluded using the criteria introduced in Section 2.3. It is readily seen 

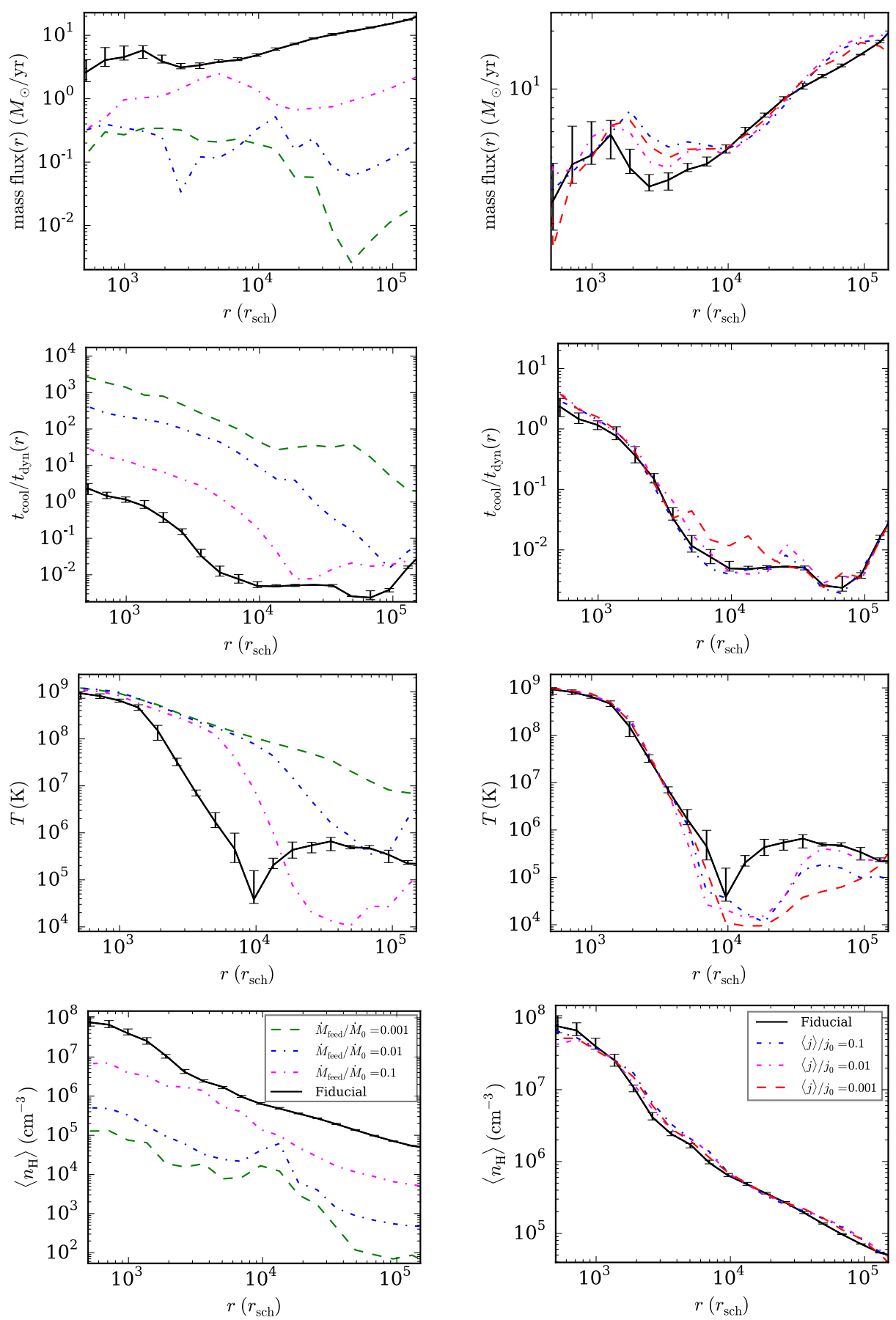

Fig. 3.- The top row shows the radial profiles of mass flux (integrated over $4 \pi$ solid angle). The second row shows the bremsstrahlung cooling time normalized by the local dynamical time $t_{\text {dyn }}$. The third row shows the gas temperature. The bottom row shows the gas number density $\left\langle n_{\mathrm{H}}\right\rangle$. The left column shows comparisons among simulations with different feeding rate (Runs $0,4,5,8$ ), while the right column varies the expectation value of specific angular momentum of feeding gas at the boundary of our simulation domain (Runs $0,1,2,3$ ). All statistics are calculated by averaging through $4 \pi$ solid angle and excluding the cold disk. Each curve is the result of statistics about a single simulation run, showing the median through 100 data dumps. Error bars, presenting the 50 per cent frequency range, are only plotted for the "fiducial" simulation run. 
that the amount of mass flux (top row) getting into the region of several $10^{3} r_{\text {sch }}$ is dependent strongly on the boundary feeding rate (see the left column) and relatively weakly on the initial specific angular momentum distribution (see the right column). The higher the gas feeding rate at the boundary, the higher the amount of gas reaches the inner regions.

What has happened to the gas that does not reach the inner regions? To answer this question, we examine the second row of Figure 3, which show the cooling time as a function of radius. It is seen that, in all cases, the ratio of cooling time to dynamical time, $t_{\mathrm{cool}} / t_{\mathrm{dyn}}$, is always less than unity until the gas has reached within about $10^{4} r_{\text {sch }}$, the dominant X-ray emitting region that we see in the simulations. This re-enforces the statement that the heating process is mainly shock heating in stead of adiabatic compression, because the adiabatic heating time scale is likely to be comparable to the dynamical free-fall time scale. It is instructive to note that the cooling time of gas in the $r \sim 10^{4} r_{\text {sch }}$ regions tends to be anti-correlated with the gas feeding rate; i.e., the higher the gas feeding rate, the larger the amount of gas that enters the inner regions, and hence the shorter the cooling time. The "saturation" of X-ray luminosity with respect to feeding rate is attributed to this, reflecting that higher cooling rate prevents gas emissivity from going up linearly with feeding rate in part. Throughout most of the radial range, mass outflow is negligible in the runs with high feeding rate. However, due to the very slow speed of cooling compared to dynamical time, fluctuations of radial flow becomes important at small radii, making it impossible to obtain accurate statistics therein-especially for the runs with low feeding rate, where the radial mass flux becomes unreliable at small radii, because "apparent" inflow and outflow due to velocity fluctuations become dominant.

Using Equation 7 to define the hot coronal gas temperature as a function of radius, we find that the gas cooling time due to bremsstrahlung emission is,

$$
\begin{aligned}
t_{\mathrm{ff}} & =\frac{3 k T_{\text {vir }}}{2 n_{\mathrm{H}} \Lambda\left(T_{\mathrm{vir}}\right)} \\
& \simeq 3.8 \times 10^{9} \mathrm{~s}\left(\frac{r}{3 \times 10^{3} r_{\mathrm{sch}}}\right)^{-1 / 2}\left(\frac{n_{\mathrm{H}}}{10^{6} \mathrm{~cm}^{-3}}\right)^{-1} \\
& \simeq 17 t_{\mathrm{dyn}}\left(\frac{M_{\mathrm{BH}}}{10^{8} M_{\odot}}\right)^{-1}\left(\frac{r}{3 \times 10^{3} r_{\mathrm{sch}}}\right)^{-2}\left(\frac{n_{\mathrm{H}}}{10^{6} \mathrm{~cm}^{-3}}\right)^{-1} .
\end{aligned}
$$

Given the gas density seen in Figure 2, we can now understand the behavior of cooling time seen in the second row of Figure 3. Gas cooling time becomes longer than the dynamical time only within a radius of several times $10^{3} r_{\text {sch }}$ at a density of $n_{\mathrm{H}} \sim 10^{7} \mathrm{~cm}^{-3}$. It is informative to notice that the cooling time at $r \leq 10^{3} r_{\text {sch }}$ in all cases is longer than the dynamic time, seen in the bottom panels of Figure 3. This indicates that a significant amount of hot thermal gas of temperature $\geq 10^{9} \mathrm{~K}$ may be "sustained" for at least several local dynamical times, even in the absence of continued supply of gas (and hence energy). On the other hand, the cooling time at $r \sim 10^{3}-10^{4} r_{\text {sch }}$ is shorter than the dynamical time, suggesting that it is more "difficult" to maintain gas at temperature $\sim 10^{8-9} \mathrm{~K}$ without continued supply of gas. This cooling constraint suggests that there is a maximum possible luminosity, if one sets $t_{\mathrm{ff}}=t_{\mathrm{dyn}}$ at each radius, which in turn limits the gas density at each radius. We confirm those 
arguments by inspecting the third row in Figure 3, in which the "non-cooling" range of radius (varies with each different feeding condition) shows consistent slope (approximately $T \propto r^{-1}$ in temperature plots. Inclusion of inverse Compton processes alters gas properties in the inner regions, which will be quantified later.

We now examine how the observable properties of the X-ray emitting regions depend on the gas feeding conditions at the boundary. The top-left panel of Figure 4 shows the $X$ ray luminosity in three bands as a function of gas feeding rate at a fixed $\langle j\rangle / j_{o}$ for the oneface feeding series (solid curves) and the two-face feeding series (dashed curves). We see that the X-ray luminosity in all energy bands increase with gas feeding rate monotonically. Over the entire range examined, the increase of $X$-ray luminosity with increasing feeding rate is sublinear, indicating that the amount of gas flux reaching the central regions $\left(\leq 10^{4} r_{\mathrm{sch}}\right)$ increases at a rate slower than the gas feeding rate at the outer boundary. In addition, there is hint that the rate of increase of luminosity further flattens significantly near the high end ( $\dot{M}_{\text {feed }} \geq 20 M_{\odot} / \mathrm{yr}$ ) of the feeding rate range. This "saturation" of X-ray luminosity with respect to increasing feeding rate is attributed to the fact that higher cooling rate prevents influx gas going up linearly with feeding rate at the boundary, see in Figure 3.

What is interesting is that, in the overlapping range of $\dot{M}_{\text {feed }} \sim 1-10 M_{\odot} / \mathrm{yr}$, the two series of runs with very different $\langle j\rangle / j_{o}$ ( 0.001 for the solid curves versus 1 for the dashed curves), seen in the top-left panel of Figure 4, differ only by a factor of a few in X-ray luminosity at a given $\dot{M}_{\text {feed }}$. The bottom-left panel of Figure 4 shows X-ray luminosity as a function of $\langle j\rangle / j_{o}$ and the dependence is a bit complicated. We see that, for the one-face feeding runs, the $X$-ray luminosity increases mildly as the mean specific angular momentum of the gas fed at the boundary, $\langle j\rangle$, increases, with the same feeding rate $\dot{M}_{\text {feed, } 0}$ in all cases. One might have expected that, since a lower $\langle j\rangle$ corresponds to a larger fraction of low angular momentum

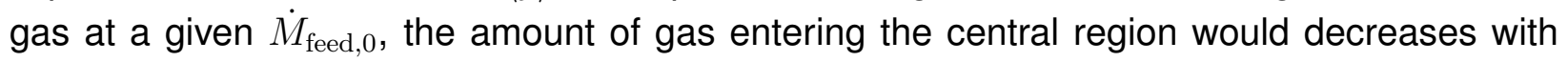
increasing $\langle j\rangle$, which is opposite to the trend, albeit mild, seen. This indicates that it is not the gas at the very low end of the initial angular momentum distribution that goes into the central regions, rather, the amount of gas actually going to the central region is a result of interactions of gas of varying initial angular momenta. On the other hand, for the two-face feeding runs, we do see that in most cases, the X-ray luminosity decrease with increasing $\langle j\rangle$. Overall, the findings suggest complexities in the gas interactions that affect the eventual angular momentum distribution at the low end, which presumably affects the amount of gas eventually entering the central, shock heated region powering the X-ray emission.

From the right panels of Figure 4. it is seen that the bremsstrahlung emission spectra are broadly peaked in the energy band $\left[10^{1} \mathrm{keV}, 10^{2} \mathrm{keV}\right]$ in general for cases with high enough feeding rate. Above $10^{2} \mathrm{keV}$, the spectra drop steeply. These spectral features are quite generic for all simulation runs, indicating that the same shock heating mechanism applies to hard X-ray emissions throughout the parameter space represented by Figure 4. For very low accretion rate $\dot{M}_{\text {feed }} / \dot{M}_{\text {feed, } 0}=0.001$, a significant steepening of the spectrum above $10 \mathrm{keV}$ is seen, indicative of inability of gas penetrating into the inner regions, which physically is 

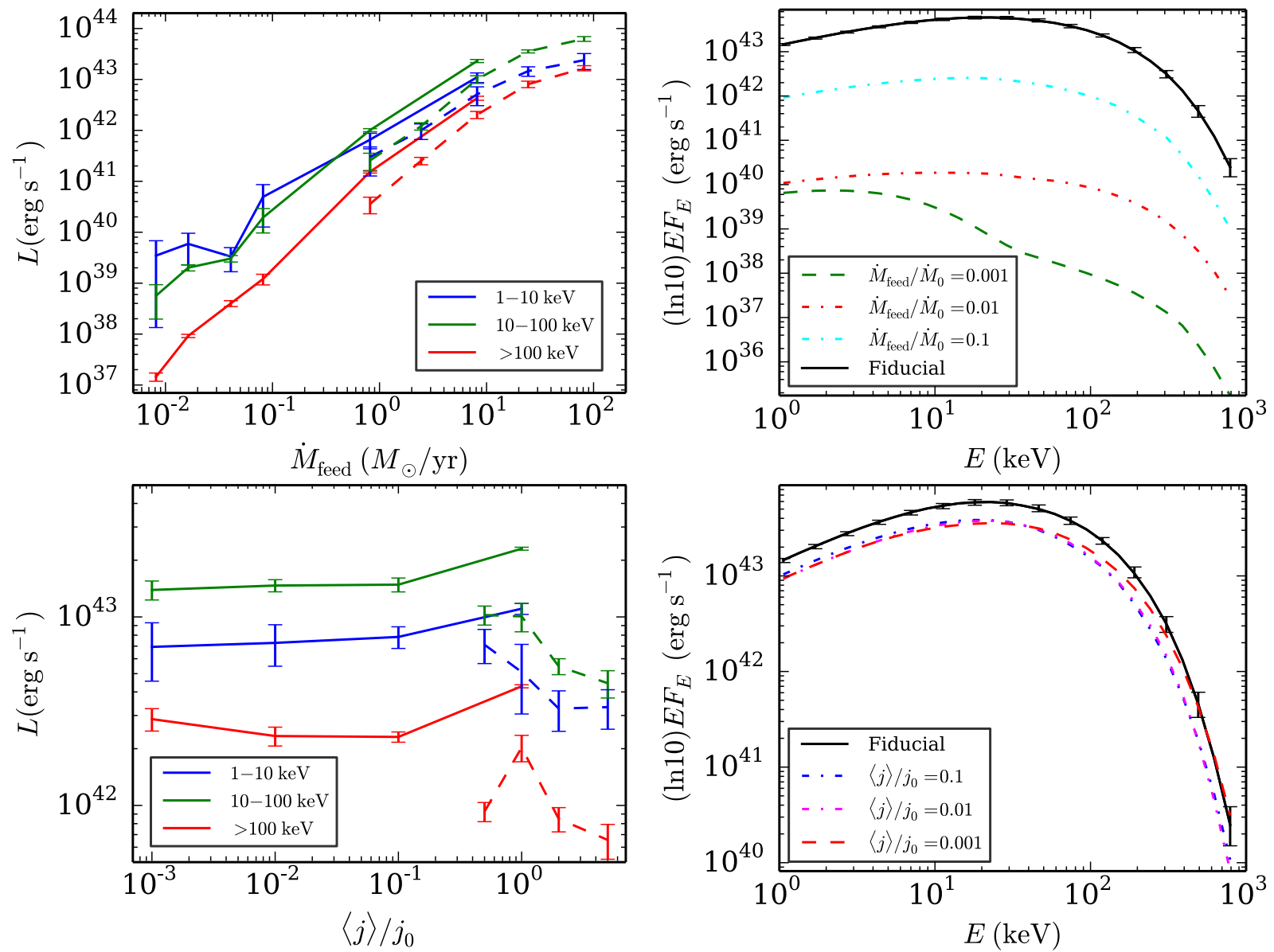

Fig. 4.- Top-left panel shows the X-ray luminosity in three bands as a function of gas feeding rate $\dot{M}_{\text {feed }} / \dot{M}_{\text {feed,0 }}$ for the one-face feeding with $\langle j\rangle / j_{o}=0.001$ (Runs 3,4,5,6) shown as solid curves and for the two-face feeding with $\langle j\rangle / j_{o}=1$ (Runs 9,10,11,12,13) shown as dashed curves. Bottom-left panel shows the X-ray luminosity in three bands as a function of the expectation value of feeding specific angular momentum $\langle j\rangle / j_{o}$ with mass feeding rate fixed at $\dot{M}_{\text {feed, } 0}$ for the one-face feeding (Runs $1,2,3,4$ ) shown as solid curves and for the two-face feeding (Runs 11,14,15,16) shown as dashed curves, at the same $\dot{M}_{\text {feed, } 0}$. Top-right panel and bottom-right panels compare the emission spectra for the $\dot{M}_{\text {feed }} / \dot{M}_{\text {feed, } 0}$ series and the $\langle j\rangle / j_{o}$ series, respectively. Each curve presents the median out of 100 snapshots with the errorbars representing the interquartile, where shown. For the spectra, we multiply each $F_{E}$ by $\ln (10) E$, so that integration of spectrum can be carried out along $E$ through the figures. 

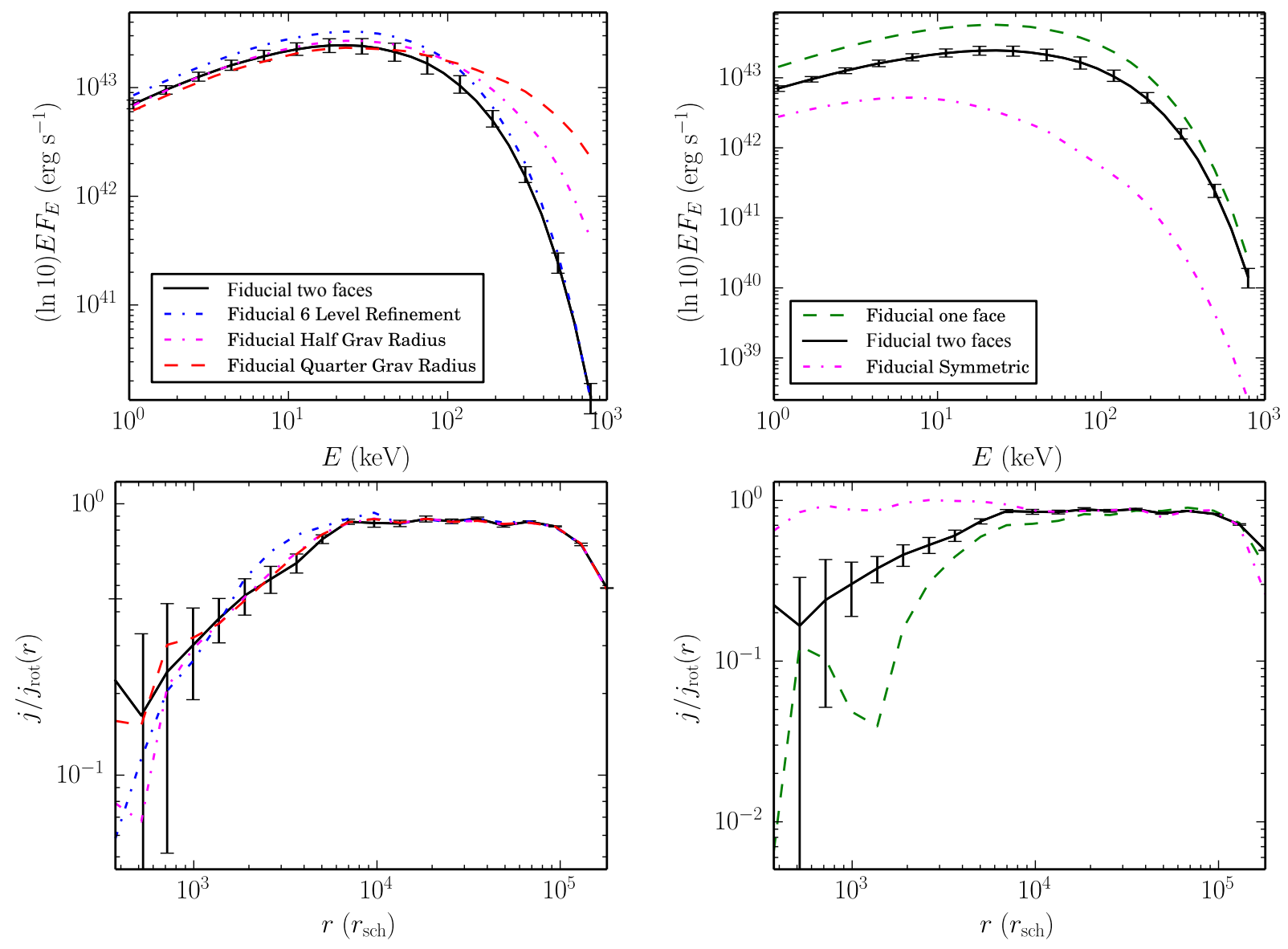

Fig. 5.- Two left panels show the radiation spectrum (upper panel) and specific angular momentum (lower panel), normalized by local circular rotation orbit specific angular momentum $j_{\text {rot }}$ ), for the resolution series (Runs $0,17,18,20$ ). Two right panels show the same results for feeding pattern series with $\dot{M}_{\text {feed,0 }}$ (Runs $0,11,19$ ). Each curve is based on 100 data dumps, with median and inter-quartiles shown only plotted for the "fiducial" simulation run. 
attributable to the increased pressure gradient in those cases. Those facts imply that feeding conditions are key factors to the X-ray bremsstrahlung emission in the vicinity of central black hole and at very low feeding rate, the X-ray spectrum due to bremsstrahlung emission softens.

Here, we further examine how results depend on the feeding patterns and also numerical resolution in Figure 5. In the upper-right panel we see that feeding the box in a "symmetric" way (i.e. via all four side faces) is actually giving considerably lower amount of X-ray radiation than the other cases, with identical total feeding rates in all cases. To provide some clue, the lower-right panel of Figure 5 shows the specific angular momentum normalized by local circular rotation orbit specific angular momentum. We see that the the "symmetric" feeding pattern is able to sustain high specific angular momentum - $j / j_{\text {rot }}$ is always close to unity - all the way down to the center. In this case, the gas that manages to inflow into the inner regions is seen to be marginally on rotation support, which in turn has posed a significant bottleneck on the amount of gas inflowing; in other words, the amount of low angular momentum gas is much smaller in this case compared to other cases and in addition more of it is less prone to shocking being on rotation support. Thus, in this case, luminosity is seen to be significantly lower and the spectrum significantly softer, peaking in the band $[1 \mathrm{keV}, 10 \mathrm{keV}]$. We see that the one-face feeding case produces a higher luminosity than the two-face feeding case, which in turn is higher than the "symmetric" feeding case. If an infalling cold stream or gas cloud gives rise to a high feeding rate episode, one might argue that in this case the "real-world" situations would be better approximated by "one face" or a fraction of a face feeding.

In terms of the softening radius $a$, the spectrum is hardened for a smaller $a$, as can be seen in Figure 5. This can be understood as follows. A smaller $a$ allows gas to continue to infall towards smaller radius and heated to higher temperature. We expect that, with still higher resolution, the spectrum at high energy end may drop off less steeply as shown in Figure 6 in the absence of other physical processes that may be operating at small radii, such as inverse Compton process, which we will discuss now.

\subsection{A Unified X-ray Emission Model Compared to Observations}

In the simulations analyzed so far, we do not include inverse Compton (IC) up-scattering of seed photons produced by the accretion disk. IC process would have two effects. On one hand, the corona gas in the central region will be cooled by the UV/optical photons emanating from the accretion disk. On the other hand, the up-scattered UV/optical photons will contribute to the X-ray emission (and affect the UV/optical spectrum as well), which, traditionally, is thought to be the primary X-ray emission process of AGNs (e.g. Walter \& Courvoisier 1992; Haardt 1993; Haardt et al. 1994). We now consider the effects of IC on gas cooling and the resulting X-ray emission, jointly with the free-free emission, in the context of the coronal gas structure learned from the simulations.

Before we integrate IC process into our simulation results, it is useful to make a di- 


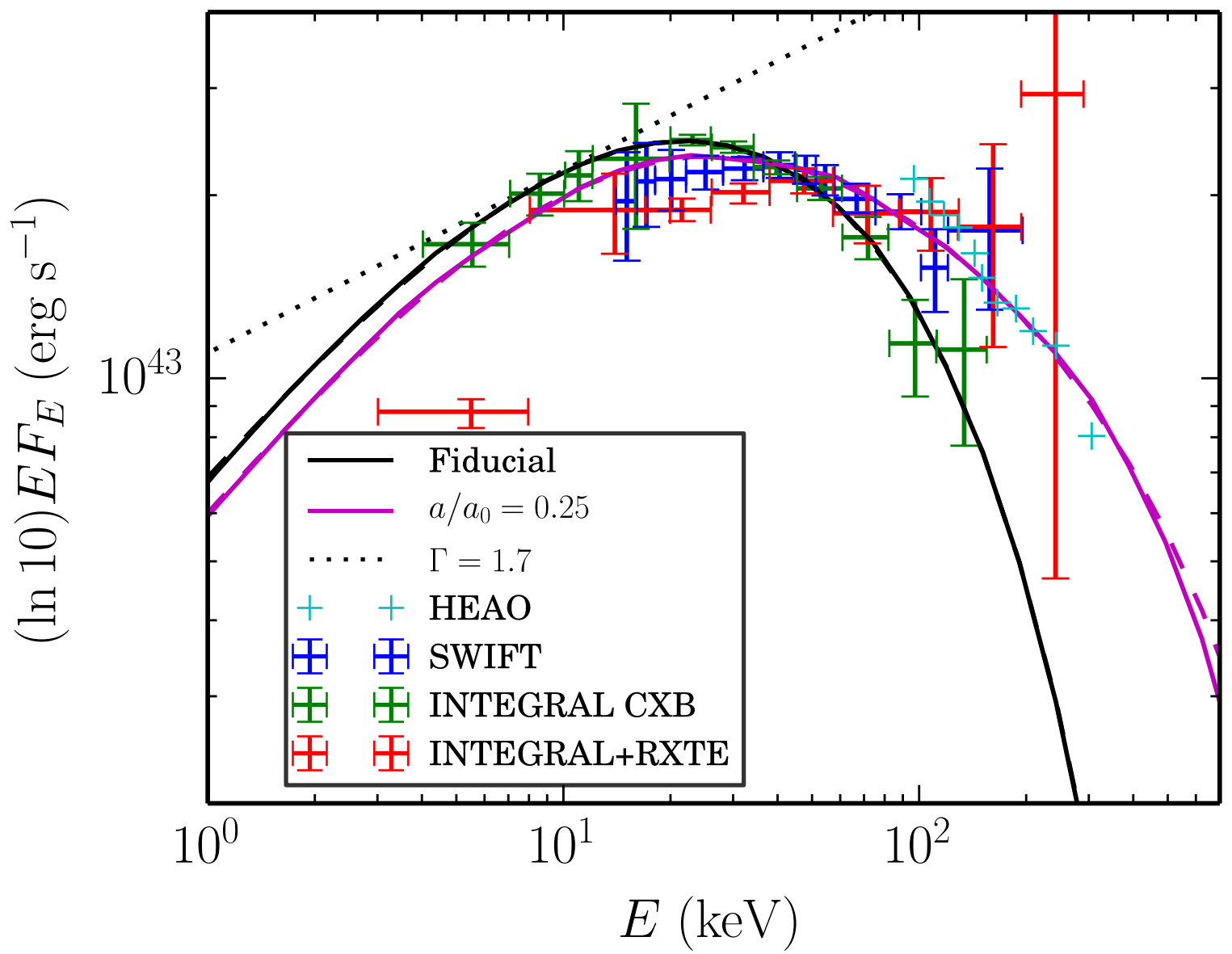

Fig. 6. - shows X-ray spectra of the simulation series with varying gravitational softening radius $a$ (the $a / a_{0}=0.5$ case lies in-between $a / a_{0}=0.25$ and $a / a_{0}=1$ and is omitted for display clarity). Dotted line added to the figure indicates a pure power-law with photon index $\Gamma=1.7$ (e.g. Pappa et al. 2001). Observation results are also overlaid onto the figure for comparison. Unobscured composite AGN spectra from SWIFT observation (see Burlon et al. 2011) are indicated by blue errorbars. Errorbars in green are the compilation of INTEGRAL X-ray background, while those in red are for composite AGN spectra observed by INTEGRAL and RXTE combined (Sazonov et al. 2008). Data points coming from HEAO1-A4 MED shown by " + " in cyan without errorbars (Kinzer et al. 1997). 
rect comparison between X-ray observations and simulation results without IC contributions, shown in Figure 6. We clarify that the computed spectra (curves in Figure 6) is a composite bremsstrahlung emission from shock heated gas of varying temperatures, primarily in the central $10^{4} r_{\text {sch }}$ region, without any contribution from the gas disk. We see in Figure 6 that, at the soft energy end in the $[1 \mathrm{keV}, 5 \mathrm{keV}]$ range, the simulated spectrum has too hard a slope. Specifically, we find a spectral index of 1.31 in this energy range, which is to be compared with the observed canonical value of $[0.1 \mathrm{keV}, 0.3 \mathrm{keV}]$, ranging from $\sim 1.5$ to $\sim 2$, (e.g. Pappa et al. 2001; Gallagher et al. 2002; Falocco et al. 2012) from stacked spectra in the $[1 \mathrm{keV}, 10 \mathrm{keV}]$ band. We expect that inclusion of metal line emission (e.g. Cen et al. 1995; Brocksopp et al. 2006) and contribution from the accretion disk will likely soften the spectra, perhaps bringing the results into better agreement with observations. Thus, since this energy range is not the focus of our study, we are not worried about with this apparent discrepancy at this time.

In the hard X-ray range of $[5 \mathrm{keV}, 300 \mathrm{keV}]$ that is our focus, the simulated spectrum provides a good match to observations. Both the broad peak of the spectrum at $[10 \mathrm{keV}, 30 \mathrm{keV}]$ and the hard X-ray spectral shape at $[30 \mathrm{keV}, 300 \mathrm{keV}]$ are in good agreement with observations, including the hard X-ray background that is dominated by AGNs (Kinzer et al. 1997; Sazonov et al. 2008; Burlon et al. 2011). Given our completely un-fine-tuned simulation metrics, it is non-trivial to achieve such a good agreement between generic simulations and observations. This is strongly indicative that the overall $X$-ray emission of all AGNs as an population can be largely accounted for by bremsstrahlung emission. Also implied by this observation is that the overall hard X-ray background can be largely accounted from contribution of high UV/optical luminosity AGNs.

To understand and account for the overall variety of X-ray spectra of AGNs, inverse Compton processes may be indispensable, as we show now. In order to take IC processes into account, it is necessary to extrapolate our simulation results of density and temperature to smaller radii, in part because of resolution limitations and in part because of neglect of IC processes in the simulations. Based on Figures 2 and 3, and the discussions in Appendix A, we conclude that, in the regions where hard X-ray emission is dominated by bremsstrahlung, density may be approximated by the scaling $\rho \propto r^{-7 / 4}$, and temperature as $T \propto r^{-1}$. Due to the lack of treatment of Compton cooling in the simulations, we cannot expect those scaling relations of "non-conservative" quantities, such as temperature and density, to still hold in the IC cooling dominated region when properly treated. We thus define the power-law indices $\alpha$ and $\beta$, for temperature and density respectively, and leave both adjustable in the IC dominated region and the density profile in the bremsstrahlung dominated region $\eta$ also adjustable,

$$
T \propto\left\{\begin{array} { l l } 
{ r ^ { - 1 } , } & { r > r _ { \text { tran } } , } \\
{ r ^ { - \alpha } , } & { r \leq r _ { \text { tran } } ; }
\end{array} \quad \rho \propto \left\{\begin{array}{ll}
r^{-\eta}, & r>r_{\text {tran }}, \\
r^{-\beta}, & r \leq r_{\text {tran }} .
\end{array}\right.\right.
$$

Based on the discussion given in Appendix A.3, at low $L_{\mathrm{UV}}$, we use $\alpha=1$, while $\alpha+\beta \leq 3 / 2$ for high $L_{\mathrm{UV}}$ cases. Also, in Appendix A.1, we derive that $\eta \simeq 7 / 4$ for the situations with low 
feeding rate. We note that the profile introduced by equation (11) is applied to extrapolate the simulation into the central coronal region, where inverse Compton process possibly contributes significantly to the overall X-ray emission of individual AGNs, especially those with low accretion rates, as we will elaborate in the rest of this subsection.

The value of bremsstrahlung-inverse Compton transition radius $r_{\text {tran }}$ is defined in Equation (A11) in the Appendix A. For normalization at $r>r_{\text {tran }}$ outside the IC dominant region, we use the virial temperature for $T$ as in equation (7) and electron density $n_{e}$ as (both of which are consistent with simulations)

$$
n_{e}=n_{0}\left(\frac{r}{3 \times 10^{3} r_{\text {sch }}}\right)^{-\eta}, \quad r>r_{\text {tran }} .
$$

We adjust the normalization of the power-law relations in the $r \leq r_{\operatorname{tran}}$ region, so that the $T$ and $n_{e}$ functions are continuous at $r=r_{\text {tran }}$. Also, a "cap" temperature is assigned based on Equation (A25) in the IC dominated region, considering cooling and heating balance.

The density and temperature profiles are applied to calculate the hard X-ray spectra, using a code that we have developed and thoroughly tested to compute IC scattering and resultant spectra from first-principle Monte Carlo simulations (see Appendix B for details). This threedimensional IC scattering code, called ICode, is to be made publicly available immediately with access information provided in Appendix B. For the UV source from disk emission, we adopt single-temperature black body spectra for simplicity without loss of essential physical features and observable output, whose peak of distribution in photon energy is adjustable but is set to be $15 \mathrm{eV}$ (e.g. Haardt \& Maraschi 1993) for our calculation presented. The source is designed as a standard thin disk, whose inner edge is set to be consistent with the UV luminosity (see Tomsick et al. 2009). At each radius on the disk, the rate of the number of photons emitted per unit area is in accordance with the effective temperature profile standard $\alpha$-disk model (e.g. Shakura \& Sunyaev 1973). In some cases where the AGN UV/optical luminosity emanating from the disk is high, $r_{\text {tran }}$ may encroach into the bremsstrahlung regions in current simulations. For those cases, we also calculate the bremsstrahlung spectra analytically with analytically obtained density and temperature profiles. We will also explore the impact of the value of $n_{0}$ defined in Equation (12).

The density of shock heated gas in the central region, $n_{6} \equiv n_{0} /\left(10^{6} \mathrm{~cm}^{-3}\right)$ at $3 \times 10^{3} r_{\text {sch }}$, depends on the feeding rate and angular momentum of gas at the outer boundary. Here we will express gas feeding rate $\dot{M}_{\text {feed }}$ in terms of $\dot{M}_{\text {Edd }}$, the "Eddington" feeding rate for the SMBH, defined as,

$$
\dot{M}_{\mathrm{Edd}}=\frac{L_{\mathrm{Edd}}}{\alpha_{\mathrm{rad}} c^{2}},
$$

where $\alpha_{\text {rad }} \equiv L /\left(\dot{M} c^{2}\right)$ is the radiative efficiency. Typically, $\alpha_{\text {rad }} \simeq 0.1$, which gives $\dot{M}_{\text {Edd }} \simeq$ $2.2 M_{\odot} /$ yr for $M_{\mathrm{BH}}=10^{8} M_{\odot}$. In Figure 7, we show how $n_{6}$ varies with different $\langle j\rangle$ and $\dot{M}_{\text {feed }}$. It is seen that, at a given, relatively low $\dot{M}_{\text {feed }}, n_{6}$ does not sensitively depend on $\langle j\rangle$, as seen earlier in Figure 4 . On the other hand, $n_{6}$ increases with increasing $\dot{M}_{\text {feed }}$, albeit with a rate 


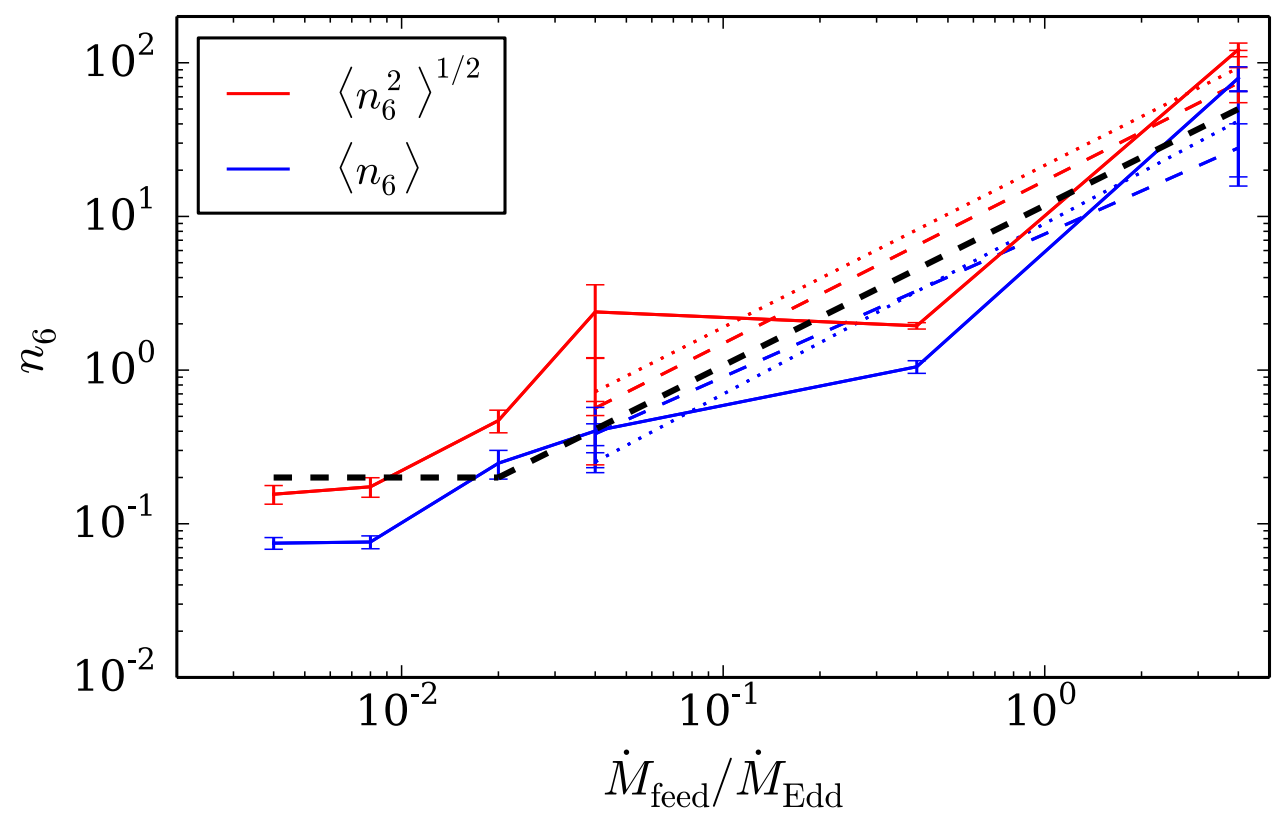

Fig. 7.- Shows the dependence of $n_{6}$ on feeding gas feeding rate and angular momentum $\langle j\rangle / j_{0}$. The solid lines have $\langle j\rangle / j_{0}=10^{-3}$, dotted lines have $\langle j\rangle / j_{0}=10^{-2}$, and dashed lines have $\langle j\rangle / j_{0}=10^{-1}$. The blue lines show $n_{6}$ that is volume averaged, while the red line is the root-mean-squared value of $n_{6}$. Errorbars represent the interquartiles. The black heavy dashed line indicates the overall trend of $n_{6}$ 's feeding rate dependency.

that is sublinear. At higher $\dot{M}_{\text {feed }} / \dot{M}_{\text {Edd }}$ beyond the range shown, we find that $n_{6}$ flattens out and we attribute the high-end "saturation" of $n_{6}$ to quadratically-increasing cooling rate with density, which causes progressively more "dropout" of coronal materials. At the low end of $\dot{M}_{\text {feed }} / \dot{M}_{\text {Edd }}\left(\lesssim 4 \times 10^{-3}\right)$, it is seen that $n_{6}$ also flatten out. This is because cooling time starts to significantly exceed the dynamical time (see also Equation 10 and A15) such that a hot corona may be "maintained" nearly independent of $\dot{M}_{\text {feed }}$ at low $\dot{M}_{\text {feed }}$ end. With the results in Figure 7, if we express $L_{\mathrm{UV}} / L_{\mathrm{Edd}} \simeq\left(\alpha_{\text {rad }} / 0.1\right) \dot{M}_{\text {feed }} / \dot{M}_{\mathrm{Edd}}$, one then obtains a relatively constrained range of $n_{6}$ for a given $L_{\mathrm{UV}} / L_{\text {Edd }}$. Typically, in the regime that $\dot{M}_{\text {feed }} / \dot{M}_{\text {Edd }} \gtrsim 10^{-1}$, $n_{6}$ is seen to be $\sim 10^{0}$; below $\dot{M}_{\text {feed }} / \dot{M}_{\text {Edd }} \gtrsim 10^{-1}, n_{6}$ slowly approaches $\sim 10^{-1}$.

To show the versatility and capability of our model, we use four observed systems spanning a wide range in spectral shape (data are from Vasudevan \& Fabian 2009). In our model, the temperature of black body source of seed photons from the disk is set by observations according to Vasudevan \& Fabian (2009). In this case, we simply vary $\alpha$ and $\beta$ manually until we arrive at a reasonable fit. The results obtained are based on detailed IC scattering processes using ICode. It is noted that our calculations of IC processes are performed in three dimensions, albeit with the spherically symmetric geometry for the IC scattering region and the disk geometry for the UV emitting regions in this case. The fitting process also ensures that the Eddington ratio and the luminosity in $[2 \mathrm{keV}, 10 \mathrm{keV}]$ both match those of the observed system in question. Since our model is constructed based on simulation results and physical 

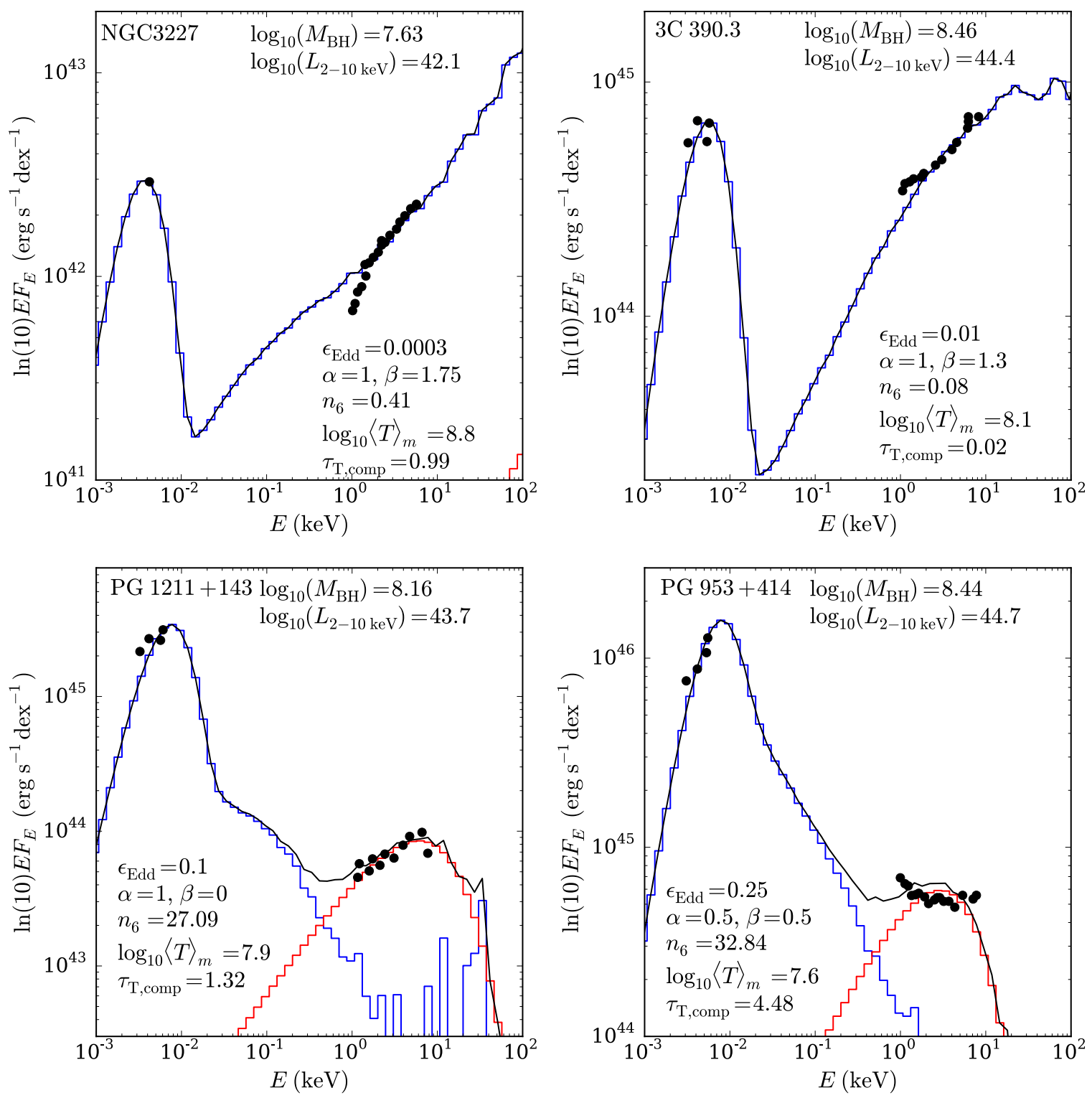

Fig. 8.- Composite UV and X-ray spectra, consisting of original UV, inverse Compton and bremsstrahlung components. In each panel, the original UV and inverse Compton X-ray component generated by Monte Carlo simulation is shown in blue stepped curve; analytically computed bremsstrahlung contribution is indicated by red stepped curve. The total spectral intensity, which is the sum of blue and red curves, is shown by the black curve. In each case, the information for the observed object (object name, SMBH mass and $\mathrm{X}$-ray luminosity in the $[2 \mathrm{keV}, 10 \mathrm{keV}]$ band) is indicated at the top of the panel, while the input model parameters (Eddington ratio for the disk luminosity, temperature profile slope $\alpha$, density profile slope $\beta$, normalization density $n_{0}$ ) are indicated in lower part of the panel. The derived parameters (total radially integrated IC optical depth $\tau_{\text {IC }}$ and optical depth-weighted temperature of the IC region $\left\langle T_{\mathrm{IC}}\right\rangle_{\tau}$ ) are also indicated for those cases whose $\mathrm{X}$-ray emission is dominated by IC. The observed data points (without errorbars) are shown as dots. 
constraints, the arrived set of model parameters is physically attainable.

Detailed comparisons are shown in Figure 8. The top two panels show two cases with hard spectral shape, where the hard X-ray luminosity is comparable to, or exceeds, that of the UV bump, whereas the bottom two panels show two cases with "normal" X-ray to UV luminosity ratio in the range $1-10 \%$. Three points are worth noting. First of all, our physical model can easily accommodate the observed variety of spectral shapes. Second, comparing the top panels with the bottom panels, we see a trend: the relative IC contribution to hard $\mathrm{X}$-ray emission increases, as the UV luminosity from the disk decreases. The physical reason for this, at zero-th order, is that a low UV radiation bath allows for the existence of an enlarged IC region (i.e., a larger IC optical depth) and a higher mean temperature in the IC region. The IC optical depth determines the probability distribution function of the number of scatterings (including multiple scattering events), while the temperature determines the energetics (i.e., energetic gain of photons) of scattering events. Third, in a physically attractive sense, our "self-consistent", coupled treatment of IC processes and central UV source distinguishes itself from the usual model with the assignment of the putative hot corona that is separate from the property of the central UV source. This removes the arbitrariness of the hand-picked coronal gas and places physically attainable models within a limited parameter space.

Let us now go beyond just anecdotal evidence, by comparing with observations statistically and systematically. To do that, we use $\alpha_{\text {ox }}$, the optical to X-ray spectral index, with the "standard" definition (e.g. Lusso et al. 2010) of

$$
\alpha_{\mathrm{ox}} \equiv-\frac{\log _{10}\left(L_{2 \mathrm{keV}} / L_{2500}\right)}{2.605} .
$$

We construct our theoretical models with two different sets of power-law indices: $(\alpha, \beta)=$ $(1,1.2)$ (dashed lines in Figure 9), and $(\alpha, \beta)=(1,0.5)$ (solid lines in Figure 9). The chosen values of $n_{0} \equiv n_{6} \times 10^{6} \mathrm{~cm}^{-3}$ span the range found in Figure 7 . For a given $n_{6}$, we only vary the Eddington ratio $\epsilon_{\mathrm{Edd}}$ of the UV photon source.

Figure 9 shows our theoretical optical to X-ray spectral index $\alpha_{\text {ox }}$ as a function of optical/UV luminosity $\left[\nu L_{\nu}(2500) / L_{\text {Edd }}\right]$ for five different values of $n_{6} \equiv n_{0} /\left(10^{6} \mathrm{~cm}^{-3}\right)$. Comparing the four theoretical curves in Figure 9, as expected, as $n_{6}$ increases, with all other parameters fixed, $\alpha_{\mathrm{ox}}$ decreases (i.e., spectrum becomes harder) at a given Eddington ratio (for UV/optical luminosity). This is because a higher $n_{6}$ gives rise to a higher IC optical depth hence a larger hard X-ray luminosity. Along each curve, as the Eddington ratio (for UV/optical luminosity) increases, $\alpha_{\text {ox }}$ increases (i.e., spectrum becomes softer). This is because a higher Eddington ratio reduces the IC region hence a lower IC optical depth and more importantly a lower temperature of the IC region, in combination resulting in a lower X-ray luminosity. Intriguingly and perhaps profoundly, this simple model with a reasonable range of $n_{0}$ that is consistent with Figure 9) can account for the observed data points exceedingly well.

More specifically, the trend of our model curves going from lower left to upper right result in, naturally, two "deserts" on the upper-left and lower-right corners, seen in the observational 


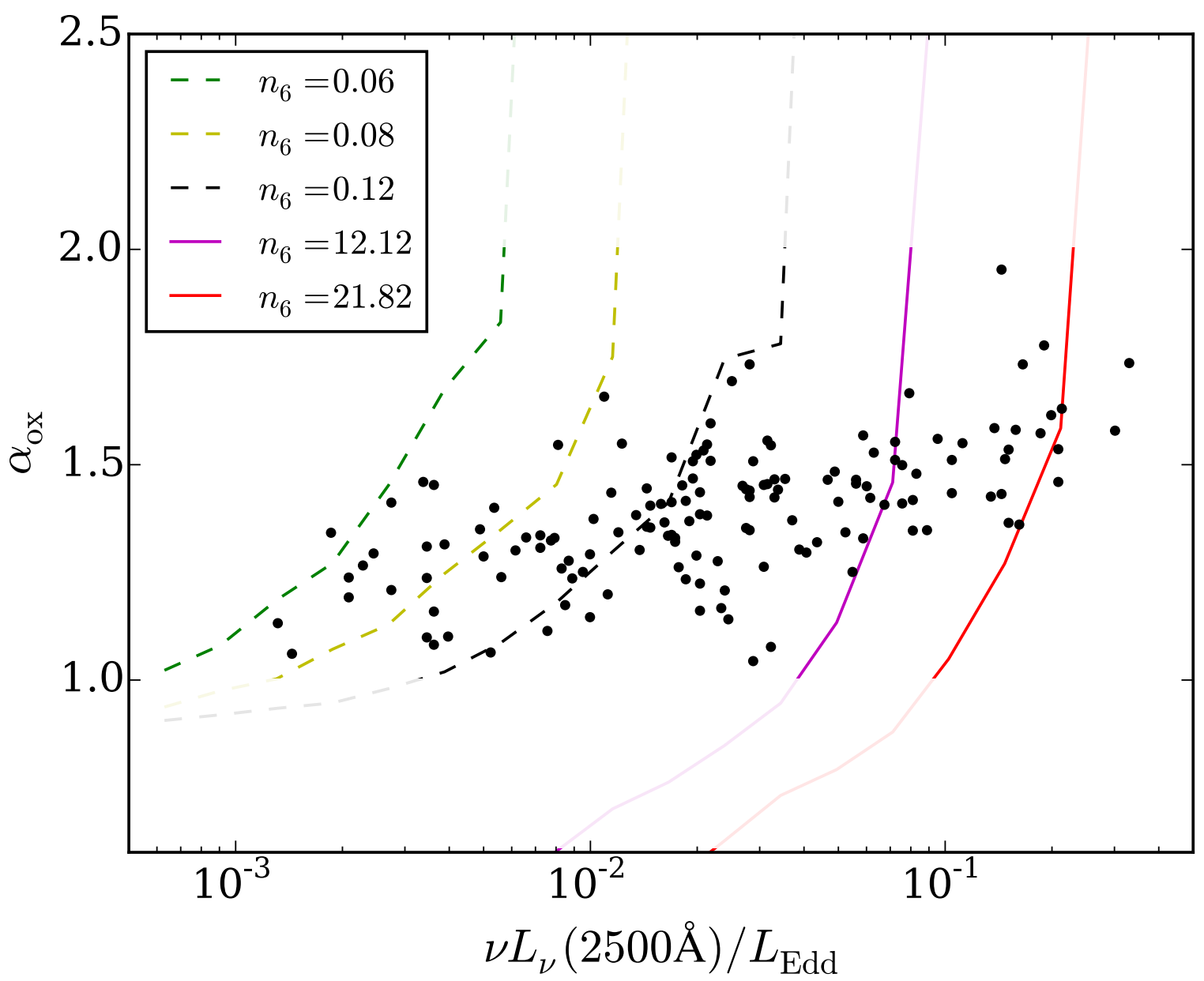

Fig. 9.- Shows our theoretical optical to X-ray spectral index $\alpha_{\mathrm{ox}}$ as a function of optical/UV luminosity $\left[\nu L_{\nu}(2500) / L_{\text {Edd }}\right]$ for five different values of $n_{6} \equiv n_{0} /\left(10^{6} \mathrm{~cm}^{-3}\right)$. Two sequences are shown: dashed lines have $\alpha=1$ and $\beta=1.2$, and solid lines $\alpha=1$ and $\beta=0.5$. To compare reasonably, we convert the luminosity of both theoretical and observational data into $\nu L_{\nu}(2500) / L_{\mathrm{Edd}}$, which is a dimensionless that already takes the variation of observed $M_{\mathrm{BH}}$ into account. Observational data points, shown as black points, are from Lusso et al. (2010). The regions that are not plausible in terms of parameter space constraint due to the correlation between $n_{6}$ and UV luminosity (i.e., a narrow range of $n_{6}$ at a given UV luminosity) shown in Figure 9 are shown as lower and upper "faint" segments of each line.

data. If the model prediction for the desert in the upper-left corner holds up, the implication is that a lower value of $n_{6} \sim 0.1$ is required, as our simulations suggest, with small but non-negligible AGN activities of $\nu L_{\nu}(2500) / L_{\mathrm{Edd}} \geq 10^{-3}$ fed by a commensurate gas inflow. Similarly, if the model prediction for the desert in the lower-right corner is correct, it would imply an upper limit on $n_{6} \sim 30$, which may either be accounted for due to cooling saturation or limitations on gas inflow gas around SMBHs, also suggested by our simulations. These two limits are consistent with the examples seen in Figure 7. Moreover, the range of observed $\alpha_{\mathrm{ox}} \sim 1-2$ is naturally explained due to the correlation between $n_{6}$ and UV luminosity, a narrow range of 
$n_{6}$ at a given UV luminosity, shown in Figure 9. We note that there are some degeneracies between $n_{0}$ and the choices of temperature and density profiles. Nonetheless, our model is being set apart from other models in its physical simplicity, a multitude of predictive power and falsifiability.

\section{Discussion and Conclusions}

We perform three-dimensional hydrodynamic simulations, covering the spatial domain $\sim 10^{2} r_{\text {sch }}$ to $2 \mathrm{pc}$ around the central supermassive black hole of mass $10^{8} M_{\odot}$, with detailed radiative cooling processes and gravity. Analysis shows that, for a realistic range of gas feeding rates from large scales ( $\sim 2 \mathrm{pc}$ ), gravitational shock heating is the dominant heating process, resulting in a significant amount of high temperature gas in the inner $\leq 10^{4} r_{\text {sch }}$ coronal region above the accretion disk with its radiative cooling time scale exceeding gravitational heating time scale. We show that the composite bremsstrahlung emission spectrum due to coronal gas of various temperatures from our generic simulations are in reasonable agreement with the overall ensemble spectrum of AGNs and hard X-ray background in the $[5 \mathrm{keV}, 300 \mathrm{keV}]$ range. This indicates that most of the hard $X$-ray radiation from AGNs can be accounted for by the bremsstrahlung emission of the gravitationally shock heated coronal gas.

We then combine the simulation results with a post-simulation analysis that includes a treatment of the inverse Compton processes (up-scattering of soft UV photons produced by the accretion disk), using a newly developed code from first-principle Monte Carlo simulations, (see Appendix B for details of our newly developed code that is made publicly available). One of the most attractive features of our model is that the hot coronal gas in the central inverse Compton region is a significantly constrained and integrated part of the gas feeding process, rather than an ad hoc separate component. Further, the property (density and temperature radial profiles) of the gas in the inverse Compton region is dependent on the luminosity of the accretion disk. We show that the combined modeling can readily account for the wide variety of AGN spectral shape, which can be understood physically in simple ways. One natural outcome, one of the most salient features of our model, is an anti-correlation between SMBH accretion disk luminosity and spectral hardness: as the luminosity of SMBH accretion disk decreases, the hard X-ray luminosity increases relative to the UV/optical luminosity. This is because, as the luminosity of SMBH accretion disk decreases, the radius of the region whose electrons are not cooled by UV photons decreases, resulting in an increase in the energy of scattering electrons. We show that this general trend not only is borne out in individual observed AGNs but also explains the spectral hardness-Eddington ratio relation observed. Our model suggests two "deserts" of AGNs, with either low-luminosity and soft spectral index, or high-luminosity and hard spectral index, that may be verifiable. Moreover, the range of observed $\alpha_{\text {ox }} \sim 1-2$ is naturally explained due to a relative tight correlation between the coronal gas density in the central region and AGN UV luminosity. 
Computing resources were in part provided by the NASA High- End Computing (HEC) Program through the NASA Advanced Supercomputing (NAS) Division at Ames Research Center. The research is supported in part by NSF grant AST-1108700 and NASA grant NNX12AF91G.

\section{A. Scaling Relations and Constraints of Physical Quantities}

We start our derivations by assuming a steady state. Consider a thin spherical shell at radius $r$ with thickness $\delta r$, and volume $4 \pi r^{2} \delta r$. At the outer and inner boundaries of this shell, mass and energy flow in and out, respectively. In general, within the shell, there are influx and outflux of mass, along with some material cooling and "dropping out" from the hot phase (to join the disk). Energetically, thermal energy is injected by gravitational acceleration and subsequent thermalization, along with advected thermal energy and cooling. In a steady state, the net change of both mass and energy within the shell is zero.

\section{A.1. Energy Balance and Scaling Relations in Bremsstrahlung Dominated Region}

In this subsection, we derive the scaling relation of density and temperature in the region where the dominating cooling mechanism is bremsstrahlung.

We denote the hot gas "drop-out" rate as $(\mathrm{d} \dot{M} / \mathrm{d} r)_{\mathrm{drop}}$. Conservation of mass in this shell says,

$$
0=\frac{\Delta M}{\mathrm{~d} t}=\dot{M}(r+\delta r)-\dot{M}(r)-\left(\frac{\mathrm{d} \dot{M}}{\mathrm{~d} r}\right)_{\mathrm{drop}} \delta r=\left[\frac{\mathrm{d} \dot{M}}{\mathrm{~d} r}-\left(\frac{\mathrm{d} \dot{M}}{\mathrm{~d} r}\right)_{\mathrm{drop}}\right] \delta r,
$$

which then gives the net mass flux through the boundaries equal to the drop-out rate,

$$
\frac{\mathrm{d} \dot{M}}{\mathrm{~d} r}=\left(\frac{\mathrm{d} \dot{M}}{\mathrm{~d} r}\right)_{\text {drop }} .
$$

Next, we consider the energy budget within the radial shell, where its temporal change has three different components:

$$
\Delta E=\Delta E_{\text {flow }}+\Delta E_{\text {grav }}+\Delta E_{\text {rad }},
$$

where $\Delta E_{\text {flow }}$ is the amount of energy that travels with mass flow, $\Delta E_{\text {grav }}$ represents gravitational heating, and $\Delta E_{\text {rad }}$ accounts for the radiative cooling. The $\Delta E_{\text {flow }}$ term can be expressed in terms of temperature gradient using Equation (A2) as,

$$
\frac{\Delta E_{\text {flow }}}{\mathrm{d} t}=\frac{3}{2} T(r+\delta r) \frac{\dot{M}(r+\delta r)}{\mu m_{p}}-\frac{3}{2} k T(r) \frac{\dot{M}(r)}{\mu m_{p}}-\frac{3 k T(r)}{2 \mu m_{p}}\left(\frac{\mathrm{d} \dot{M}}{\mathrm{~d} r}\right)_{\mathrm{drop}} \delta r=\frac{3 k}{\mu m_{p}} \dot{M}(r) \frac{\mathrm{d} T}{\mathrm{~d} r} \delta r .
$$


For the gravitational heating component, we express it in terms of mass flux,

$$
\Delta E_{\text {grav }}=\left[\left(\frac{G M_{\mathrm{BH}}}{r^{2}}\right) 4 \pi r^{2} \delta r \rho(r)\right]\left(\left|\left\langle v_{r}\right\rangle\right| \mathrm{d} t\right)=\left(\frac{G M_{\mathrm{BH}}}{r^{2}}\right) \dot{M} \delta r \mathrm{~d} t .
$$

where we use $\dot{M}(r)=4 \pi r^{2} \rho\left|\left\langle v_{r}\right\rangle\right|$, and $\left\langle v_{r}\right\rangle$ is the mass-weighted radial velocity of the shell.

Based on the bremsstrahlung emissivity per unit frequency in Equation (5) and using the Gaunt factor equal to unity, the total bremsstrahlung emissivity is obtained by an integration with respect to $\nu$ is, (e.g., Draine 2011)

$$
\epsilon_{\mathrm{ff}}=\int_{0}^{\infty} \mathrm{d} \nu j_{\mathrm{ff}} \simeq C_{\mathrm{ff}} \rho^{2} T^{1 / 2} .
$$

Here $C_{\mathrm{ff}}$ is a constant. We note that the Gaunt factor correction is negligible for the integrated emissivity $\epsilon_{\mathrm{ff}}$ in hard X-ray emitting plasma (e.g. the correction factor is only 1.09 for a $10^{9} \mathrm{~K}=$ $86 \mathrm{keV} / \mathrm{k}$ gas). Hence, the component of bremsstrahlung energy loss is,

$$
\left(\frac{\Delta E_{\mathrm{rad}}}{\mathrm{d} t}\right)_{\mathrm{ff}}=-4 \pi r^{2} \epsilon_{\mathrm{fff}} \delta r=-4 \pi r^{2} C_{\mathrm{ff}} \rho^{2} T^{1 / 2} \delta r .
$$

Combining Equations (A4), (A5) and (A7) with Equation (A3), we obtain,

$$
0=\frac{\Delta E}{\mathrm{~d} t \delta r}=\frac{3 k}{2 \mu m_{p}} \dot{M} \frac{\mathrm{d} T}{\mathrm{~d} r}+\frac{G M_{\mathrm{BH}}}{r^{2}} \dot{M}-4 \pi r^{2} C_{\mathrm{ff}} \rho^{2} T^{1 / 2} .
$$

Using this relation and Equation (7), (A8) becomes

$$
r^{2} \rho^{2} T^{1 / 2} \propto \dot{M} r^{-2} ; \quad \rho \propto\left(r^{-3} T^{-1 / 2}\right)^{1 / 2} \propto r^{-7 / 4+\gamma / 2} .
$$

We utilize the results found in our simulations to constrain $\gamma$ in $\dot{M}(r) \propto r^{\gamma}$. We find that $\gamma \simeq 0$ at the low feeding rate limit, which yields $\beta=7 / 4$. At at high feeding rate limit, $\gamma \simeq 1 / 2$, yielding $\beta=3 / 2$. We plot the density profile for the hot $\left(T>10^{6} \mathrm{~K}\right)$ gas in the inner region both arithmetic average and root-mean-squared average through $4 \pi$ solid angle, in Figure 10. Compared with the guiding line showing $\beta=7 / 4$ slope, the density profile in the central regions agrees nicely with our derivations.

\section{A.2. Transition from Bremsstrahlung to Inverse Compton}

We now derive the expression of another important quantity, the optical depth of the central inverse Compton region. We define the "transition radius", $r_{\text {tran }}$, such that inverse Compton cooling dominates at $r<r_{\text {tran }}$ and bremsstrahlung cooling dominates at $r>r_{\text {tran }}$, and they are equal at $r_{\text {tran }}$. It is easily seen that, using $\eta=7 / 4$, in terms of $r$, the ratio between $t_{\mathrm{IC}}$ and $t_{\mathrm{ff}}$ varies as,

$$
\frac{t_{\mathrm{IC}}}{t_{\mathrm{ff}}} \propto \frac{r^{2}}{r^{5 / 4}} \propto r^{3 / 4}
$$



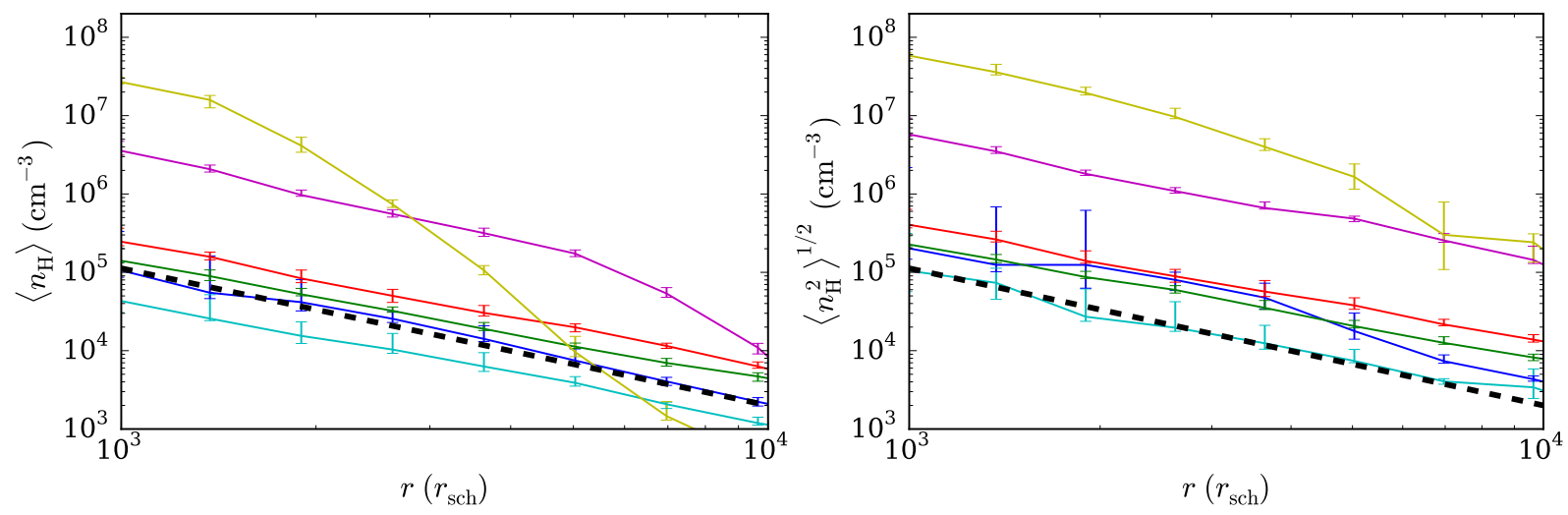

Fig. 10.- Shows density (left panel: $\left\langle n_{\mathrm{H}}\right\rangle$; right panel: $\left\langle n_{\mathrm{H}}^{2}\right\rangle^{1 / 2}$ ) profile for runs with different feeding rates $\dot{M}_{\text {feed }} / \dot{M}_{\text {feed, } 0}$ (cyan: 0.001, Run 8; blue: 0.002, Run 7; green: 0.005, Run 6; red: 0.01 , Run 5; magenta: 0.1 , Run 4; yellow: 1 , Run 3). The heavy dashed line shows the power index $\beta=7 / 4$.

Setting $t_{\mathrm{IC}} / t_{\mathrm{ff}}$ to unity, we obtain,

$$
r_{\text {tran }} \simeq 3 \times 10^{7} r_{\text {sch }}\left(\frac{n_{0}}{10^{6} \mathrm{~cm}^{-3}}\right)^{-4 / 3}\left(\frac{M_{\mathrm{BH}}}{10^{8} M_{\odot}}\right)^{4 / 3} \epsilon_{\mathrm{Edd}}^{4 / 3} .
$$

In Figure 11, we illustrate the comparison between inverse Compton and analytically extrapolated free-free cooling time scales, under different luminosity $\left(\epsilon_{\mathrm{Edd}}\right)$ and feeding $\left(n_{6}\right)$ conditions. With each combination of parameters, the value of $r_{\text {tran }}$ is illustrated by a crossing point of an inverse Compton (blue dashed) line and a free-free (black solid) line.

This directly leads to the Thomson optical depth of inverse Compton region, assuming a $n \propto r^{-\beta}$ density profile,

$$
\begin{aligned}
\tau_{\mathrm{T}, \mathrm{IC}} & \simeq \sigma_{\mathrm{T}} n_{0}\left(\frac{r_{\mathrm{tran}}}{3 \times 10^{3} r_{\mathrm{sch}}}\right)^{-7 / 4} r_{\mathrm{tran}} \int_{r_{\mathrm{sch}}}^{r_{\text {tran }}}\left(\frac{r}{r_{\text {tran }}}\right)^{-\beta} \frac{\mathrm{d} r}{r_{\text {tran }}} \\
& =0.0033 \epsilon_{\mathrm{Edd}}^{-1}\left(\frac{M_{\mathrm{BH}}}{10^{8} M_{\odot}}\right)^{-1}\left(\frac{n_{0}}{10^{6} \mathrm{~cm}^{-3}}\right) \times \begin{cases}\ln \left(\frac{r_{\text {tran }}}{r_{\mathrm{sch}}}\right), & \beta=1 ; \\
\frac{1}{1-\beta}\left[1-\left(\frac{r_{\mathrm{sch}}}{r_{\text {tran }}}\right)^{1-\beta}\right], & \beta \neq 1 .\end{cases}
\end{aligned}
$$

Note the anti-correlation between $\tau_{\mathrm{T}, \mathrm{IC}}$ and $\epsilon_{\mathrm{Edd}}$.

\section{A.3. Constraints and Energy Balance in Inverse Compton Region}

Here we derive the scaling relations in the innermost coronal region, where cooling process is dominated by inverse Compton. Under the assumption of a steady state, there are two different types, depending on the feeding/inflow rate and gas density. 


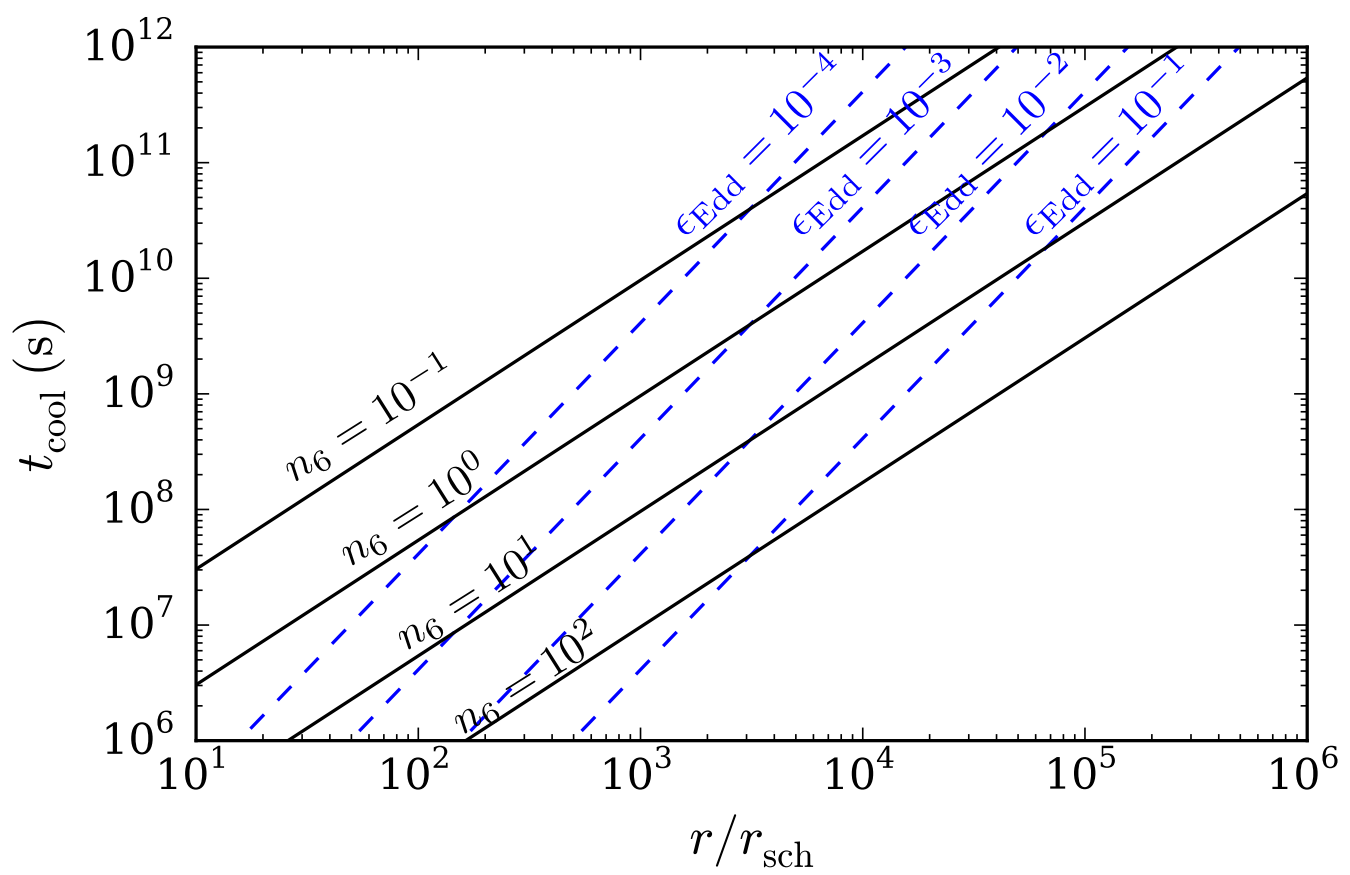

Fig. 11.- Compares inverse Compton cooling time [blue dashed lines; see equation (A15)] and free-free cooling time [black solid lines, see equation (10)] as functions of radius. $\eta=7 / 4$ is taken here for radial density profile, and other necessary parameters are labeled in the figure near each line.

In the first type, gas feeding/inflow rate is low and gas density in the central region correspondingly low. In this case, the gas cooling time is longer than dynamical time, and hence the gas "turnover" (i.e., cooling off) rate is low. Thus, the gas may be considered to be in quasi-hydrostatic equilibrium. The coronal gas in quasi-hydrostatic equilibrium in the spherical symmetry case reads,

$$
-\frac{G M_{\mathrm{BH}}}{r^{2}}+\frac{1}{\rho} \frac{\mathrm{d} p}{\mathrm{~d} r}=0 ; \quad \frac{1}{\rho} \frac{\mathrm{d}(\rho T)}{\mathrm{d} r} \propto r^{-2} .
$$

Assuming power-law density and temperature profiles specified in equation (11), we insert $\alpha$ and $\beta$ into equation (A13), to result in

$$
r^{\beta} \frac{\mathrm{d}}{\mathrm{d} r} r^{-(\alpha+\beta)} \propto r^{-2} ; \quad \alpha=1 .
$$

The power-law index for the density profile, under the quasi-hydrostatic equilibrium condition, is not constrained, due to the domination of SMBH gravity over coronal gas self-gravity.

In the second type, gas feeding/inflow rate is high and gas density in the central region correspondingly high. In this case, the gas cooling time is shorter than dynamical time, and hence the gas is in constant flux. Although the steady state assumption may still be valid, the gas is far from being in quasi-hydrostatic equilibrium. For this regime, we constrain the power-law profiles as follows. 
For a parcel of hot electrons bathed in a radiation field of energy density $u_{\mathrm{ph}}$, the cooling time of the electrons due to IC processes is (e.g. Rybicki \& Lightman 1979)

$$
\begin{aligned}
t_{\mathrm{IC}} & =\frac{3}{8} \frac{m_{e} c}{\sigma_{T}} \frac{1}{u_{\mathrm{ph}}} \\
& =3.7 \times 10^{7} \mathrm{~s}\left(\frac{M_{\mathrm{BH}}}{10^{8} M_{\odot}}\right)\left(\frac{\epsilon_{\mathrm{Edd}}}{0.1}\right)^{-1}\left(\frac{r}{3 \times 10^{3} r_{\mathrm{sch}}}\right)^{2},
\end{aligned}
$$

where $m_{e}$ is the electron mass, $c$ the speed of light, $\sigma_{T}$ the Thompson cross section, $\epsilon_{\mathrm{Edd}} \equiv$ $L_{\mathrm{UV}} / L_{\mathrm{Edd}}$ the Eddington ratio for the disk luminosity. One important feature to note is that $t_{\mathrm{IC}}$ decreases with decreasing radius $r$, implying a tendency to produce hard X-ray spectrum via IC processes. Using Equation (A15), we deduce the scaling relation of energy loss rate due to inverse Compton (the dependencies on $M_{\mathrm{BH}}$ and $\epsilon_{\mathrm{Edd}}$ are absorbed into the factor $C_{\mathrm{IC}}$, which is independent of radius)

$$
\epsilon_{\mathrm{IC}}=C_{\mathrm{IC}} \rho T r^{-2}
$$

Using the same arguments used earlier in the derivation for the bremsstrahlung region (§A.1), we obtain the radiative energy loss term

$$
\left(\frac{\Delta E_{\mathrm{rad}}}{\mathrm{d} t}\right)_{\mathrm{IC}}=-4 \pi r^{2} \epsilon_{\mathrm{IC}} \delta r=-4 \pi C_{\mathrm{IC}} \rho T \delta r,
$$

which, when combined with Equations (A3), (A4) (A5) gives

$$
\rho \propto T^{-1}\left(\frac{3 k}{2 \mu m_{p}} \frac{\mathrm{d} T}{\mathrm{~d} r}+\frac{G M_{\mathrm{BH}}}{r^{2}}\right) \dot{M} ; \quad \rho T \propto \dot{M} r^{-2} .
$$

If we further assume that the relation $\dot{M} \propto r^{1 / 2}$ (for high feeding rate) still holds in the inverse Compton domain, we have

$$
\alpha+\beta=3 / 2
$$

It is reasonable to assume that the simulation results without IC cooling may be extrapolated into the IC cooling region in the high gas feeding rate regime, because cooling is important and gas dynamics is primarily determined by gravity and hydrodynamics, not primarily by thermodynamics. In any case, since IC cooling becomes more important at smaller radii, which in turn may induce, relatively, more gas drop-out at smaller radii, it is therefore likely that $\alpha+\beta \leq 3 / 2$ holds in realistic situations.

We utilize the inverse Compton cooling and gravitational heating processes to provide further constraints. For a fluid element of mass $\delta m$ falling towards the central black hole at virial velocity, the gravitational heating rate is

$$
\left(\frac{\Delta E}{\mathrm{~d} t}\right)_{\text {grav }}=F_{\text {grav }}\left(\frac{\mathrm{d} r}{\mathrm{~d} t}\right)_{\text {virial }} \leq \frac{G M_{\mathrm{BH}} \delta m}{r^{2}}\left(\frac{G M_{\mathrm{BH}}}{r}\right)^{1 / 2}=\frac{\left(G M_{\mathrm{BH}}\right)^{3 / 2}}{r^{5 / 2}} \delta m .
$$

Plugging in various numbers, we have the energy gain rate per unit mass due to gravitational heating

$$
\left(\frac{\Delta E}{\delta m \mathrm{~d} t}\right)_{\text {grav }} \leq 1.02 \times 10^{10} \mathrm{erg} \mathrm{s}^{-1} \mathrm{~g}^{-1}\left(\frac{M_{\mathrm{BH}}}{10^{8} M_{\odot}}\right)^{-1}\left(\frac{r}{10^{3} r_{\mathrm{sch}}}\right)^{-5 / 2}
$$


In the mean time, the amount of energy that is removed from this fluid element per unit time is,

$$
\left(-\frac{\Delta E}{\mathrm{~d} t}\right)_{\mathrm{IC}} \simeq\left(\frac{3 k T \delta m}{2 m_{p}}\right)\left(\frac{3 m_{e} c}{8 \sigma_{\mathrm{T}}} \frac{4 \pi r^{2} c}{L_{\mathrm{Edd}} \varepsilon_{\mathrm{Edd}}}\right)^{-1}
$$

Inserting the numbers, we get the inverse Compton energy loss rate per unit mass,

$$
\left(-\frac{\Delta E}{\delta m \mathrm{~d} t}\right)_{\mathrm{IC}}=3.08 \times 10^{11} \mathrm{erg} \mathrm{s}^{-1} \mathrm{~g}^{-1} \epsilon_{\mathrm{Edd}}\left(\frac{M_{\mathrm{BH}}}{10^{8} M_{\odot}}\right)^{-1}\left(\frac{T}{10^{9} \mathrm{~K}}\right)\left(\frac{r}{10^{3} r_{\mathrm{sch}}}\right)^{-2} \text {. }
$$

The ratio is

$$
\left(\frac{\Delta E}{\delta m \mathrm{~d} t}\right)_{\text {grav }} /\left(-\frac{\Delta E}{\delta m \mathrm{~d} t}\right)_{\mathrm{IC}} \leq 0.033 \varepsilon_{\text {Edd }}^{-1}\left(\frac{r}{10^{3} r_{\mathrm{sch}}}\right)^{-1 / 2}\left(\frac{T}{10^{9} \mathrm{~K}}\right)^{-1} .
$$

This result yields an upper limit on temperature in the inverse Compton region as a function of Eddington ratio $\epsilon_{\mathrm{Edd}}$ and radius $r$ by setting the ratio to unity:

$$
T_{\text {max }, \text { IC }} \lesssim 1.1 \times 10^{8} \mathrm{~K}\left(\frac{\epsilon_{\mathrm{Edd}}}{0.1}\right)^{-1}\left(\frac{r}{3 \times 10^{3} r_{\mathrm{sch}}}\right)^{-1 / 2}
$$

\section{B. ICode: A Monte Carlo Code for Inverse Compton Scattering Processes}

In order to compute the spectra taking into account the inverse Compton scattering processes, we have developed a Monte Carlo code in $\mathrm{C}++$ with OpenMP shared-memory parallelization, called "ICode". ICode follows directly the transport and scattering processes of photons from first principles. ICode is constructed with modular structures and allows for easy modifications for physical conditions, such as spatial density and temperature structures of the scattering plasma or the geometry of the sources of the soft seed photons. We emphasize that ICode does not require any simplification of the geometry of the scattering medium or photon sources and can handle arbitrary geometry for both. We properly treat all relevant regimes, including the "tran-relativistic" high-energy electrons as well as photons with energy comparable to electron's rest energy. We now describe the basic physical steps used to construct ICode.

As a photon is emitted by the soft photon source, its four-momentum is assigned in such a way that its energy obeys the spectral distribution of the source (such as a single- or multitemperature Planck distribution) and its direction is random (we assume that the source is isotropic everywhere, so that the spatial component of photon's initial four-momentum is uniformly distributed across the $4 \pi$ solid angle).

Before each scattering, a random number $\tau$ that obeys exponential distribution with unitary parameter [i.e. $\tau \sim P_{\exp }(\tau ; 1)=\mathrm{e}^{-\tau}$ ] is generated. This $\tau$ is actually the "optical depth" that this photon travels through before it is scattered by a high-energy electron. Starting from an initial point $\mathbf{x}_{0}$, this photon "walks" along the chosen direction of momentum, until the cumulative optical depth

$$
\tau\left(\mathbf{x}_{1}, \mathbf{x}_{0}\right) \equiv \int_{\mathbf{x}_{0}}^{\mathbf{x}_{1}}|\mathrm{~d} \mathbf{x}| \sigma_{\mathrm{KN}}\left(\frac{h \nu}{m_{e} c^{2}}\right) n_{e}(\mathbf{x})
$$


reaches the desired $\tau$ (that we just generated randomly following the prescribed distribution) at some $\mathbf{x}_{1}$, where the photon is scattered by an electron. In Equation (B1), $n_{e}(\mathbf{x})$ is the electron number density profile of the hot plasma, and $\sigma_{\mathrm{KN}}$ is the Klein-Nishina total cross section for photon-electron scattering (e.g. Blumenthal \& Gould 1970; Srednicki 2007)

$$
\begin{aligned}
\sigma_{\mathrm{KN}}(x) & =\frac{3}{8} \sigma_{\mathrm{T}} f_{\mathrm{KN}}(x), \quad x \equiv \frac{h \nu}{m_{e} c^{2}} \\
f_{\mathrm{KN}}(x) & \equiv\left\{\frac{2[x(x+1)(x+8)+2]}{x^{2}(2 x+1)^{2}}+\left(\frac{x^{2}-2 x-2}{x^{3}}\right) \ln (2 x+1)\right\} .
\end{aligned}
$$

Since there may be cases where electron thermal energy is comparable to $m_{e} c^{2}$ (hence upscattered photons with energy close to $m_{e} c^{2}$ are generated), it is desirable to include KleinNishina correction to account for second or higher order inverse Compton scatterings. The value of $h \nu$ is exactly the 0th component of the photon's four-momentum.

At point $\mathbf{x}_{1}$, the Lorentz factor $\gamma$ of an electron that interacts with the photon is another random variable, which obeys the relativistic Maxwellian distribution, taking the local electron temperature $T_{e}$ as an argument (e.g. Haardt 1993),

$$
\gamma \sim P_{\mathrm{e}}\left(\gamma, \Theta_{e}\right)=\frac{1}{\Theta_{e} K_{2}\left(1 / \Theta_{e}\right)} \gamma\left(\gamma^{2}-1\right)^{1 / 2} \exp \left(-\frac{\gamma}{\Theta_{e}}\right) ; \quad \Theta_{e} \equiv \frac{k T_{e}}{m_{e} c^{2}} .
$$

Here, $K_{2}$ is the second-order modified Bessel function of the second kind. The direction of that electron's spatial momentum is randomly, uniformly distributed over the $4 \pi$ solid angle.

The most convenient way to deal with scatterings is to adopt the appropriate differential cross section in the "target rest frame", i.e., the "electron rest frame" (ERF for short), in which the pre-scattering electron is at rest at the origin. Our numerical schemes follows the following steps, starting from the lab frame (LF for short):

1. Use a proper Lorentz transformation $\Lambda_{\nu}^{\mu}$ to obtain four-momentum of the electron and photon in ERF;

2. Spatially rotate the system in ERF so that the incident photon is going along the $x$-axis;

3. Use the normalized proper differential cross section and the Compton scattering formula to obtain the photon's post-scattering four-momentum in the rotated ERF;

4. Spatially rotate the system back to obtain the photon's momentum in the original ERF;

5. Use the inverse Lorentz transformation to obtain the photon's four-momentum after scattering in LF.

We denote the pre-scattering four-momentum of the electron in the LF, to be decomposed, as (following the standard of special relativity, the symbol " $\asymp$ " means "such a vector/tensor can be represented by such a matrix"),

$$
p_{e}^{\mu} \asymp \gamma m_{e} c\left(1, \beta_{x}, \beta_{y}, \beta_{z}\right)^{\mathrm{T}},
$$


where $\beta_{x}=v_{x} / c$ is the relativistic $\beta$ along $x$-axis. The Lorentz transformation in Step 1 is written as a function of $\boldsymbol{\beta} \equiv\left(\beta_{x}, \beta_{y}, \beta_{z}\right)$,

$$
\Lambda_{\nu}^{\mu}(\boldsymbol{\beta}) \asymp\left[\begin{array}{cccc}
\gamma & -\gamma \beta_{x} & -\gamma \beta_{y} & -\gamma \beta_{z} \\
-\gamma \beta_{x} & 1+(\gamma-1) \frac{\beta_{x}^{2}}{\beta^{2}} & (\gamma-1) \frac{\beta_{x} \beta_{y}}{\beta^{2}} & (\gamma-1) \frac{\beta_{x} \beta_{z}}{\beta^{2}} \\
-\gamma \beta_{y} & (\gamma-1) \frac{\beta_{x} \beta_{y}}{\beta^{2}} & 1+(\gamma-1) \frac{\beta_{y}^{2}}{\beta^{2}} & (\gamma-1) \frac{\beta_{y} \beta_{z}}{\beta^{2}} \\
-\gamma \beta_{z} & (\gamma-1) \frac{\beta_{x} \beta_{z}}{\beta^{2}} & (\gamma-1) \frac{\beta_{y} \beta_{z}}{\beta^{2}} & 1+(\gamma-1) \frac{\beta_{z}^{2}}{\beta^{2}}
\end{array}\right] .
$$

It is straightforward to verify that the inverse Lorentz transformation needed in Step 5 can be obtained by $\Lambda_{\nu}^{\dagger \mu}=\Lambda_{\nu}^{\mu}(-\boldsymbol{\beta})$. For the spatial rotation in Step 4, the rotation matrix $R_{\nu}^{\mu}$ for the four-vector consists of two sequential Eulerian rotations (the Einstein convention of summation is used hereafter),

$$
\begin{aligned}
& R_{\nu}^{\mu}(\Theta, \Phi)=T_{\rho}^{\mu}(\Phi) S_{\nu}^{\rho}(\Theta) ; \quad T_{\rho}^{\mu}(\Phi) \asymp\left[\begin{array}{rrrr}
1 & & & \\
& \cos \Phi & -\sin \Phi & \\
& \sin \Phi & \cos \Phi & \\
& & & 1
\end{array}\right] ;
\end{aligned}
$$

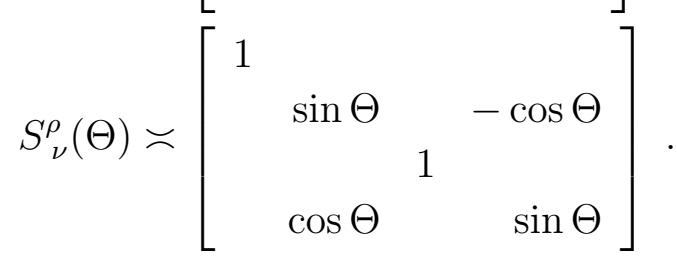

Here $\Theta$ and $\Phi$ are the direction cosines of pre-scattering photon in ERF-they are defined in such a way that the pre-scattering four-momentum of the photon in ERF is written as (its pre-scattering energy in ERF is denoted as $h \nu$ ),

$$
p_{\mathrm{ph}}^{\prime \mu} \asymp h \nu(1, \sin \Theta \cos \Phi, \sin \Theta \sin \Phi, \cos \Theta)^{\mathrm{T}} .
$$

The inverse rotation matrix needed in Step 2 is obtained by $R_{\nu}^{-1 \mu}(\Theta, \Phi)=S_{\nu}^{-1 \rho}(\Theta) T_{\rho}^{-1 \mu}(\Phi)$, and the inverses of $S$ and $T$ tensors can be obtained by transposing their corresponding matrices in Equation (B6).

For Step 3, the scattering cosine $\mu=\cos \theta$ ( $\theta$ is the scattering angle) of the post-scattering photon is (yet) another random variable that obeys the normalized Klein-Nishina distribution function,

$$
\mu \sim P_{\mathrm{KN}}\left(\mu ; \frac{h \nu}{m_{e} c^{2}}\right),
$$

whose expression reads (see also Blumenthal \& Gould 1970; Srednicki 2007),

$$
P_{\mathrm{KN}}\left(\mu ; \frac{h \nu}{m_{e} c^{2}}\right)=\left[f_{\mathrm{KN}}\left(\frac{h \nu}{m_{e} c^{2}}\right)\right]^{-1} P_{\mathrm{IC}}^{2}(\nu, \mu)\left[P_{\mathrm{IC}}(\nu, \mu)+\frac{1}{P_{\mathrm{IC}}(\nu, \mu)}-1+\mu^{2}\right] .
$$

Here, $f_{\mathrm{KN}}$ is defined in Equation (B2), and $P_{\mathrm{IC}}$ is the Compton factor, as a function of incident photon frequency $\nu$ and the scattering cosine $\mu$,

$$
P(\nu, \mu) \equiv\left[1+\frac{h \nu}{m_{e} c^{2}}(1-\mu)\right]^{-1}
$$



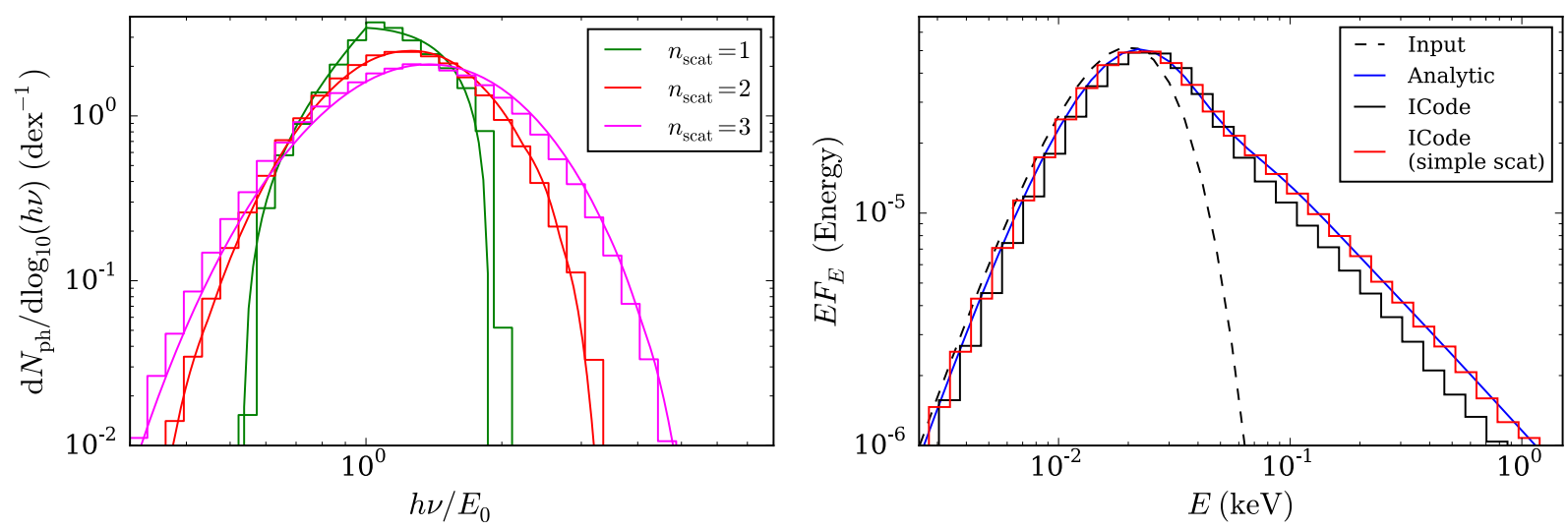

Fig. 12.- Left panel compares ICode simulated inverse Compton photon energy distribution shown as step curves with the analytic approximation results shown as smooth curves based on Rybicki \& Lightman (1979), for one (green curves), two (red curves) and three (magenta curves) consecutive scatterings. The initial photon energy is $E_{0}=51 \mathrm{eV}$ and electrons have single energy of $25.6 \mathrm{keV}(\gamma=1.05)$. Right panel presents the comparison in a more "realistic" situation, in which photons have initial energy as a black-body spectrum at $T=5.11 \mathrm{eV} / \mathrm{k}$ (black dashed curve), injected at the center of a spherical cloud at constant electron temperature $T_{e}=0.1 m_{e} c^{2} / k=5.93 \times 10^{8} \mathrm{~K}$ and homogeneous electron density $n_{e}=6 \times 10^{6} \mathrm{~cm}^{-3}$, with radius $r=0.04$ pc. Analytic result based on Rybicki \& Lightman (1979) and Haardt (1993) is plotted in blue smooth curve. Monte Carlo results are presented in step curves, for ordinary scattering model (black curve) and "simple mode" scattering model (red curve), respectively.

which is the post- to pre-scattering photon energy ratio, $P_{\mathrm{IC}}\left(\nu_{0}, \mu\right)=\nu_{1} / \nu_{0}$.

With all those steps taken, a photon is scattered to and travels along a new spatial direction that it just acquired. Then the above steps are repeated for the scattered photon, until it leaves eventually the interaction region, at which point it is collected and binned to obtain the inverse Compton spectra.

We also introduce a "simple mode" to our code, where the outcome of a single inverse Compton scattering is evaluated via the approximated formula given in e.g. Rybicki \& Lightman (1979). This mode ignores such major complications as electron recoil and anisotropic cross section, which is a good approximation in the low-energy regime.

We have thoroughly tested ICode step by step. We will just present two non-trivial tests to demonstrate the verification of the code. The comparison between results from ICode and analytic calculations is shown in Figure 12.

In the first test problem, shown in the left panel of Figure 12, we inject single-energy photon at $E_{0}=51 \mathrm{eV}$ and let the ensemble of photons experience 1, 2 and 3 inverse Compton scatterings, respectively, by $\gamma=1.05$ (i.e. kinetic energy $25.6 \mathrm{keV}$ ) single-energy electrons, and compare the results to analytic calculations based on the approximate procedures in Rybicki \& Lightman (1979). This is a strong test on the "units" used throughout our code, since 
the outcome of Compton scatterings is not convolved with electron or photon energy distribution functions. There is a small discrepancy in the Monte Carlo results tend to be slightly softer for higher order scatterings compared to the approximated analytic results, which we attribute to the inaccuracy introduced in the approximate procedure. Overall, the agreement is excellent.

In the second problem, illustrated by the right panel in Figure 12, we put the code to a more complicated test, where a photon source is embedded at the center of an isothermal $\left(T_{e}=0.1 m_{e} c^{2} / k=5.93 \times 10^{8} \mathrm{~K}\right)$ and homogeneous $\left(n_{e}=6 \times 10^{6} \mathrm{~cm}^{-3}\right)$ spherical $(r=$ $0.04 \mathrm{pc}$ ) cloud. The Thompson optical depth from the center to the surface of the cloud is 0.49 . Injected photons have a black-body spectrum at temperature $T=5.11 \mathrm{eV} / k$. This test case resembles one of the typical situations that we might encounter when dealing with AGN corona models. For comparison, analytic results are obtained based on the method used in Haardt (1993). The agreement is nearly perfect. As discussed above, the small discrepancy between "simple-mode" and "ordinary-mode" (actually used in ICode) is attributed to the inaccuracy of simplified treatment of inverse Compton processes in the "simple-mode", which becomes more severe when photons are scattered multiple times. In this test, some photons are scattered multiple times, which is manifested by the high-end power-law-like tail of the resulting X-ray photons shown. In our test, we do not artificially limit the number of scatterings that a single photon may encounter.

Given quite thorough tests done, including but not limited to the two shown above, we conclude that the ICode is ready to be distributed to the community. This ICode is publicly available at https://github. com/w11745881210/MCIC. Please cite this paper if you use ICode.

\section{REFERENCES}

Blandford, R. D. 1999, in Astronomical Society of the Pacific Conference Series, Vol. 182, Galaxy Dynamics - A Rutgers Symposium, ed. D. R. Merritt, M. Valluri, \& J. A. Sellwood, $87-+$

Blumenthal, G. R., \& Gould, R. J. 1970, Reviews of Modern Physics, 42, 237

Brocksopp, C., Starling, R. L. C., Schady, P., et al. 2006, MNRAS, 366, 953

Bryan, G. L., Norman, M. L., O'Shea, B. W., et al. 2014, ApJS, 211, 19

Burlon, D., Ajello, M., Greiner, J., et al. 2011, ApJ, 728, 58

Cen, R., Kang, H., Ostriker, J. P., \& Ryu, D. 1995, ApJ, 451, 436

Di Matteo, T. 1998, MNRAS, 299, L15

Draine, B. T. 2011, Physics of the Interstellar and Intergalactic Medium, ed. Draine, B. T. 
Elvis, M. 2000, ApJ, 545, 63

Fabian, A. C., Rees, M. J., Stella, L., \& White, N. E. 1989, MNRAS, 238, 729

Falocco, S., Carrera, F. J., Corral, A., et al. 2012, A\&A, 538, A83

Gallagher, S. C., Brandt, W. N., Chartas, G., \& Garmire, G. P. 2002, ApJ, 567, 37

Haardt, F. 1993, ApJ, 413, 680

Haardt, F., \& Maraschi, L. 1991, ApJ, 380, L51

-. 1993, ApJ, 413, 507

Haardt, F., Maraschi, L., \& Ghisellini, G. 1994, ApJ, 432, L95

Haug, E. 1975, Zeitschrift Naturforschung Teil A, 30, 1546

Hopkins, P. F., \& Quataert, E. 2010, MNRAS, 407, 1529

-. 2011, MNRAS, 415, 1027

Ichimaru, S. 1977, ApJ, 214, 840

Igumenshchev, I. V., \& Abramowicz, M. A. 2000, ApJS, 130, 463

Kinzer, R. L., Jung, G. V., Gruber, D. E., et al. 1997, ApJ, 475, 361

Liu, B. F., Mineshige, S., \& Ohsuga, K. 2003, ApJ, 587, 571

Liu, B. F., Mineshige, S., \& Shibata, K. 2002, ApJ, 572, L173

Lusso, E., Comastri, A., Vignali, C., et al. 2010, A\&A, 512, A34

Mestel, L. 1963, MNRAS, 126, 553

Miller, K. A., \& Stone, J. M. 2000, ApJ, 534, 398

Narayan, R., Mahadevan, R., \& Quataert, E. 1998, in Theory of Black Hole Accretion Disks, ed. M. A. Abramowicz, G. Björnsson, \& J. E. Pringle, 148-182

Narayan, R., \& Yi, I. 1994, ApJ, 428, L13

Ohsuga, K., \& Mineshige, S. 2011, ApJ, 736, 2

Pappa, A., Stewart, G. C., Georgantopoulos, I., et al. 2001, MNRAS, 327, 499

Pietrini, P., \& Krolik, J. H. 1995, ApJ, 447, 526

Reynolds, C. S., \& Nowak, M. A. 2003, Phys. Rep., 377, 389 
Rybicki, G. B., \& Lightman, A. P. 1979, Radiative processes in astrophysics

Sazonov, S., Krivonos, R., Revnivtsev, M., Churazov, E., \& Sunyaev, R. 2008, A\&A, 482, 517

Shakura, N. I., \& Sunyaev, R. A. 1973, A\&A, 24, 337

Spitzer, L. 1962, Physics of Fully lonized Gases

Srednicki, M. 2007, Quantum Field Theory

Stone, J. M., \& Norman, M. L. 1992, ApJS, 80, 753

Sunyaev, R. A., \& Titarchuk, L. G. 1980, A\&A, 86, 121

Tanaka, Y., Nandra, K., Fabian, A. C., et al. 1995, Nature, 375, 659

Tomsick, J. A., Yamaoka, K., Corbel, S., et al. 2009, ApJ, 707, L87

Vasudevan, R. V., \& Fabian, A. C. 2009, MNRAS, 392, 1124

Veledina, A., Vurm, I., \& Poutanen, J. 2011, MNRAS, 414, 3330

Walter, R., \& Courvoisier, T. J.-L. 1992, A\&A, 258, 255 